\title{
ON THE CONSTRUCTION OF ELLIPTIC SOLUTIONS OF INTEGRABLE BIRATIONAL MAPS
}

\author{
MATTEO PETRERA, ANDREAS PFADLER, AND YURI B. SURIS \\ (WITH APPENDIX BY YURI N. FEDOROV)
}

March 4, 2016

\begin{abstract}
We present a systematic technique to find explicit solutions of birational maps, provided that these solutions are given in terms of elliptic functions. The two main ingredients are: (i) application of classical addition theorems for elliptic functions, and (ii) experimental technique to detect an algebraic curve containing a given sequence of points in a plane. These methods are applied to Kahan-Hirota-Kimura discretizations of the periodic Volterra chains with 3 and 4 particles.
\end{abstract}

\section{INTRODUCTION}

This paper is devoted to finding solutions of integrable birational maps in terms of elliptic functions. The similar task for integrable ordinary differential equations was a popular and well-developed topic in the classical mathematics of 18-th and 19-th century. We mention here one of the most famous and prototypical examples, the Euler top. Differential equations of motion of the Euler top read

$$
\left\{\begin{array}{l}
\dot{x}_{1}=c_{1} x_{2} x_{3}, \\
\dot{x}_{2}=c_{2} x_{3} x_{1}, \\
\dot{x}_{3}=c_{3} x_{1} x_{2},
\end{array}\right.
$$

with real parameters $c_{i}$. They admit two functionally independent integrals of motion: one easily checks that the following three functions are integrals:

$$
H_{1}=c_{2} x_{3}^{2}-c_{3} x_{2}^{2}, \quad H_{2}=c_{3} x_{1}^{2}-c_{1} x_{3}^{2}, \quad H_{3}=c_{1} x_{2}^{2}-c_{2} x_{1}^{2},
$$

but only two of them are functionally independent because of $c_{1} \mathrm{H}_{1}+c_{2} \mathrm{H}_{2}+c_{3} \mathrm{H}_{3}=0$. Using these integrals, one easily sees that the coordinates $x_{j}$ satisfy the following differential equations:

$$
\left\{\begin{array}{l}
\dot{x}_{1}^{2}=\left(H_{3}+c_{2} x_{1}^{2}\right)\left(c_{3} x_{1}^{2}-H_{2}\right), \\
\dot{x}_{2}^{2}=\left(H_{1}+c_{3} x_{2}^{2}\right)\left(c_{1} x_{2}^{2}-H_{3}\right), \\
\dot{x}_{3}^{2}=\left(H_{2}+c_{1} x_{3}^{2}\right)\left(c_{2} x_{3}^{2}-H_{1}\right) .
\end{array}\right.
$$

The fact that the polynomials on the right-hand sides of these equations are of degree four implies that the solutions are given by elliptic functions, as explained in the following classical theorem, see, e.g., [4].

M. Petrera, A. Pfadler, Yu. Suris: Institut für Mathematik, MA 7-2, Technische Universität Berlin, Str. des 17. Juni 136, 10623 Berlin, Germany.

E-mail: petrera@math.tu-berlin.de, andreas.pfadler@gmail.com, suris@math.tu-berlin.de. 
Theorem 1. The general solution of the scalar differential equation

$$
\dot{x}^{2}=\alpha_{0} x^{4}+4 \alpha_{1} x^{3}+6 \alpha_{2} x^{2}+4 \alpha_{3} x+\alpha_{4}, \quad \alpha_{i} \in \mathbb{C},
$$

is given by the (time shifts of the) second order elliptic function

$$
x(t)=-\frac{\alpha_{1}}{\alpha_{0}}+\zeta(u+v)-\zeta(u)-\zeta(v)=-\frac{\alpha_{1}}{\alpha_{0}}+\frac{1}{2} \frac{\wp^{\prime}(u)-\wp^{\prime}(v)}{\wp(u)-\wp(v)},
$$

where $u=\sqrt{\alpha_{0}} t$, while the point $v$ of the corresponding elliptic curve is determined by the relations

$$
\wp(v)=\frac{\alpha_{1}^{2}-\alpha_{0} \alpha_{2}}{\alpha_{0}}, \quad \wp^{\prime}(v)=\frac{\alpha_{3} \alpha_{0}^{2}-3 \alpha_{0} \alpha_{1} \alpha_{2}+2 \alpha_{1}^{3}}{\alpha_{0}^{3}} .
$$

Here the invariants of the Weierstrass $\wp$-function are given by

$$
g_{2}=\frac{\alpha_{0} \alpha_{4}-4 \alpha_{1} \alpha_{3}+3 \alpha_{2}^{2}}{\alpha_{0}^{2}}, \quad g_{3}=\frac{\alpha_{0} \alpha_{2} \alpha_{4}+2 \alpha_{1} \alpha_{2} \alpha_{3}-\alpha_{2}^{3}-\alpha_{0} \alpha_{3}^{2}-\alpha_{1}^{2} \alpha_{4}}{\alpha_{0}^{3}} .
$$

We recall that the order of an elliptic function is, by definition, the number of its zeros (or poles) in an arbitrary parallelogram of periods, counted with multiplicities. While for a mathematician of the 19-th century it was quite a routine task to derive a differential equation of the type (3) for dependent variables of integrable systems, it seems that nothing comparable to that has been developed for discrete time integrable systems which only became popular more recently, after the advent of the modern theory of integrable systems (also known as the theory of solitons). In the few number of examples, where the exact solutions of integrable maps were actually computed, the following classical result going back to L. Euler was used. (One of the first known applications of this result to the theory of discrete integrable systems is due to R. Baxter [1].)

Theorem 2. Let $P(x, \widetilde{x})$ be an irreducible symmetric biquadratic polynomial over $\mathbb{C}$. Then the algebraic curve $\mathcal{C}=\left\{(x, \widetilde{x}) \in \mathbb{C}^{2}: P(x, \widetilde{x})=0\right\}$ has genus 1 and may be parametrized as $(x, \widetilde{x})=(f(t), f(t+\delta))$, with a second order elliptic function $f$ and some shift $\delta \in \mathbb{C}$. Conversely, for an elliptic function $f$ of order 2 and for an arbitrary $\delta \in \mathbb{C}, x=f(t)$ and $\tilde{x}=f(t+\delta)$ satisfy an algebraic relation of the form $P(x, \widetilde{x})=0$, where $P$ is an irreducible symmetric biquadratic polynomial.

Concrete computations can be performed as follows. To determine invariants of the elliptic curve described by a symmetric biquadratic relation

$$
P(x, \widetilde{x})=\alpha_{0} x^{2} \widetilde{x}^{2}+\alpha_{1} x \widetilde{x}(x+\widetilde{x})+\alpha_{2}\left(x^{2}+\widetilde{x}^{2}\right)+\alpha_{3} x \widetilde{x}+\alpha_{4}(x+\widetilde{x})+\alpha_{5}=0,
$$

consider the Hamiltonian system of differential equations

$$
\dot{x}=\frac{\partial P(x, \widetilde{x})}{\partial \widetilde{x}}, \quad \dot{\tilde{x}}=-\frac{\partial P(x, \widetilde{x})}{\partial x} .
$$

Using the relation $P(x, \widetilde{x})=0$, we can eliminate either $x$ or $\widetilde{x}$ to get

$$
\dot{x}^{2}=P(x), \quad \dot{\tilde{x}}^{2}=P(\widetilde{x}),
$$

where

$$
\begin{aligned}
P(x)= & \left(\alpha_{1}^{2}-4 \alpha_{0} \alpha_{2}\right) x^{4}+\left(2 \alpha_{1} \alpha_{3}-4 \alpha_{0} \alpha_{4}-4 \alpha_{1} \alpha_{2}\right) x^{3} \\
& +\left(\alpha_{3}^{2}-4 \alpha_{0} \alpha_{5}-4 \alpha_{2}^{2}-2 \alpha_{1} \alpha_{4}\right) x^{2}+\left(2 \alpha_{3} \alpha_{4}-4 \alpha_{1} \alpha_{5}-4 \alpha_{2} \alpha_{4}\right) x+\alpha_{4}^{2}-4 \alpha_{2} \alpha_{5} .
\end{aligned}
$$


Now, Theorem 1 can be applied to differential equations (5) in order to determine $g_{2}$ and $g_{3}$ in terms of $\alpha_{i}$.

To illustrate how a biquadratic relation of the type (4) can be derived for an integrable map, we briefly remind the relevant results for the so called Hirota-Kimura discretization of the Euler top [5, 13, 14]. The HK discretization of the Euler top (1) is:

$$
\left\{\begin{array}{l}
\widetilde{x}_{1}-x_{1}=\varepsilon c_{1}\left(\widetilde{x}_{2} x_{3}+x_{2} \widetilde{x}_{3}\right), \\
\widetilde{x}_{2}-x_{2}=\varepsilon c_{2}\left(\widetilde{x}_{3} x_{1}+x_{3} \widetilde{x}_{1}\right), \\
\widetilde{x}_{3}-x_{3}=\varepsilon c_{3}\left(\widetilde{x}_{1} x_{2}+x_{1} \widetilde{x}_{2}\right) .
\end{array}\right.
$$

The map $f: x \mapsto \widetilde{x}$ is obtained by solving (6) for $\tilde{x}$, and is a birational map of degree 6 . As can be easily verified, this map possesses the following integrals of motion which are deformations of (2):

$$
H_{i}(\varepsilon)=\frac{c_{j} x_{k}^{2}-c_{k} x_{j}^{2}}{1-\varepsilon^{2} c_{j} c_{k} x_{i}^{2}} .
$$

Only two of them are independent, since

$$
c_{1} H_{1}(\varepsilon)+c_{2} H_{2}(\varepsilon)+c_{3} H_{3}(\varepsilon)+\varepsilon^{4} c_{1} c_{2} c_{3} H_{1}(\varepsilon) H_{2}(\varepsilon) H_{3}(\varepsilon)=0 .
$$

To derive biquadratic relations for the components of the discrete time Euler top, one starts by proving the following relations:

$$
\left\{\begin{array}{l}
\widetilde{x}_{2} x_{3}-x_{2} \widetilde{x}_{3}=\varepsilon H_{1}(\varepsilon)\left(\widetilde{x}_{1}+x_{1}\right), \\
\widetilde{x}_{3} x_{1}-x_{3} \widetilde{x}_{1}=\varepsilon H_{2}(\varepsilon)\left(\widetilde{x}_{3}+x_{3}\right), \\
\widetilde{x}_{1} x_{2}-x_{1} \widetilde{x}_{2}=\varepsilon H_{3}(\varepsilon)\left(\widetilde{x}_{3}+x_{3}\right) .
\end{array}\right.
$$

From eqs. (6) and (8) there follows:

$$
\left(\widetilde{x}_{i}-x_{i}\right)^{2} /\left(\varepsilon c_{i}\right)^{2}+\left(\varepsilon H_{i}(\varepsilon)\right)^{2}\left(\widetilde{x}_{i}+x_{i}\right)^{2}=2\left(\widetilde{x}_{j}^{2} x_{k}^{2}+x_{j}^{2} \widetilde{x}_{k}^{2}\right) .
$$

It remains to express $x_{j}^{2}$ and $x_{k}^{2}$ through $x_{i}^{2}$ and integrals $H_{j}(\varepsilon), H_{k}(\varepsilon)$, which results in

$$
P_{i}\left(x_{i}, \widetilde{x}_{i}\right)=\alpha_{0}^{(i)} x_{i}^{2} \widetilde{x}_{i}^{2}+\alpha_{2}^{(i)}\left(x_{i}^{2}+\widetilde{x}_{i}^{2}\right)+\alpha_{3}^{(i)} x_{i} \widetilde{x}_{i}+\alpha_{5}^{(i)}=0,
$$

where the coefficients are expressed through the integrals of motion as follows:

$$
\begin{gathered}
\alpha_{0}^{(i)}=-4 \varepsilon^{2} c_{j} c_{k}, \quad \alpha_{2}^{(i)}=\left(1+\varepsilon^{2} c_{j} H_{j}(\varepsilon)\right)\left(1-\varepsilon^{2} c_{k} H_{k}(\varepsilon)\right), \\
\alpha_{3}^{(i)}=-2\left(1-\varepsilon^{2} c_{j} H_{j}(\varepsilon)\right)\left(1+\varepsilon^{2} c_{k} H_{k}(\varepsilon)\right), \quad \alpha_{5}^{(i)}=4 \varepsilon^{2} H_{j}(\varepsilon) H_{k}(\varepsilon) .
\end{gathered}
$$

Our goal in the present paper is to propose a toolbox which allows to compute the elliptic functions expressions for solutions of integrable maps going beyond the relatively simple situation covered by Theorem 2. Our approach is based on some classical results about elliptic functions combined with rigorous symbolic computations in the spirit of experimental mathematics.

\section{OutLine OF THE METHOD}

The theoretical basis of our method is the well known classical statement that any two elliptic functions with the same periods satisfy an algebraic relation. Specifically, we have the following claim [18], which generalizes Theorem 2 . 
Theorem 3. Let $X$ and $Y$ be two elliptic functions with the same periods, of orders $n$ and $m$, respectively. Then there exists an algebraic relation of the form $P(X, Y)=0$, with an irreducible bivariate polynomial $P(X, Y)$ satisfying

$$
\operatorname{deg}_{X} P \leq m, \quad \operatorname{deg}_{Y} P \leq n, \quad \operatorname{deg} P \leq n+m .
$$

The coefficients of $P$ are unique up to multiplication with a scalar.

As a corollary, any elliptic function $X$ satisfies an algebraic differential equation, i.e., a polynomial relation between $X$ and its derivative $X^{\prime}$. Similarly, if $X=X(t)$ is an elliptic function, then $\widetilde{X}(t)=X(t+\delta)$ for an arbitrary $\varepsilon \in \mathbb{C}$ is also an elliptic function with the same periods. Hence, $X$ and $\widetilde{X}$ are connected by a polynomial relation, called addition theorem. Theorems 1, 2 illustrate these conclusions. A famous concrete example of an addition theorem is given by the Weierstrass $\wp$-function. If we take the following wellknown formula for $\wp$-functions,

$$
\wp(u+v)+\wp(u)+\wp(v)=\frac{1}{4}\left(\frac{\wp^{\prime}(u)-\wp^{\prime}(v)}{\wp(u)-\wp(v)}\right)^{2},
$$

and eliminate all derivatives via $\wp^{\prime 2}=4 \wp^{3}-g_{2} \wp-g_{3}$, we obtain

$$
\left(X Y+Y Z+Z X+\frac{g_{2}}{4}\right)^{2}-4\left(X Y Z-g_{3}\right)(X+Y+Z)=0,
$$

where $X=\wp(x), Y=\wp(y), Z=\wp(z)$, such that $x+y+z=0$. Setting $z=\delta$, we obtain the symmetric biquadratic relation between $X=\wp(x)$ and $Y=\wp(x+\delta)$.

Of course, the above relation may be of lower degree, as, for instance, for $X=\wp$ and $Y=\wp^{\prime}$ (where $n=2, m=3$, but the total degree of $P(X, Y)=Y^{2}-4 X^{3}+g_{2} X+g_{3}$ is only 3), or for an even more trivial example of $X=\wp$ and $Y=\wp^{2}$ (where $n=2, m=4$, and $P(X, Y)=Y-X^{2}$ is of bidegree $\left.(2,1)\right)$. This is a common phenomenon related to shared poles of $X$ and $Y$. If one imposes additional conditions on the poles of $X$ and $Y$, one may obtain sharper degree bounds for $P$. In particular, the following statement holds true.

Theorem 4. Let $X$ and $Y$ be two elliptic functions with the same periods, each one of order $n$ and having $n$ simple poles. If $X$ and $Y$ have $k$ poles in common, then $\operatorname{deg} P \leq 2 n-k$.

Now we are in a position to sketch the essentials of our method. Assume that we are given a birational map $f$ on the phase space $\mathbb{R}^{n}$ with coordinates $x_{i}$,

$$
x \mapsto \tilde{x}=f(x) .
$$

We want to test whether it is solvable in terms of elliptic functions and to provide an Ansatz for explicit solutions. If orbits of $f$ can be parametrized in terms of elliptic functions, then the pairs $\left(x_{i}, \widetilde{x}_{i}\right)=\left(x_{i}(t), x_{i}(t+\delta)\right)$ considered as functions of the discrete time $t \in \delta \mathbb{Z}$ will satisfy a polynomial relation of the type

$$
P\left(x_{i}, \tilde{x}_{i}\right)=0 \text {. }
$$

The degree of $P$ in $x_{i}$ and $\widetilde{x}_{i}$ will be the same and equal to the order of the elliptic function $x_{i}$.

Suppose that all components $x_{i}$ are elliptic functions. Then one could check whether they have the same periods. For this aim, one could compute the invariants $g_{2}, g_{3}$ of all the curves given by the above relations. The computation of the invariants $g_{2}, g_{3}$ may 
be accomplished using algorithms by M. van Hoeij [17], implemented in Maple algcurves package.

Assume that all $x_{i}$ are given by elliptic functions with the same periods and (for the sake of simplicity) of the same order $n$. Then one could gather additional information about the distribution of their poles and zeros. For this aim, one looks for relations of the form

$$
Q\left(x_{i}, x_{j}\right)=0 .
$$

The degree of $Q$ now tells us whether $x_{i}$ and $x_{j}$ have a number of common poles. In particular, if $\operatorname{deg} Q=2 n-k$, then $x_{i}$ and $x_{j}$ are likely to have $k$ common poles. The same relation, considered as a polynomial relation between $1 / x_{i}$ and $1 / x_{j}$, will deliver information about possible common zeros of $x_{i}$ and $x_{j}$. Similarly, one looks for the number of coincidences between the poles of $x_{i}$ and zeros of $x_{j}$ (say). Eventually, we obtain enough information about the solutions in order to fully characterize poles and zeros of $x_{i}$. On the base of this information, one finally arrives at Ansätze, which can be rigorously verified using the classical means of mathematical analysis.

In applications, formulas for the maps $f$ are often given by implicit equations of motion, like eq. (6), the explicit formulas being much more complicated and difficult to handle. Also, explicit formulas for integrals are often very messy (compare [12, 13]). Therefore, one cannot expect to be able to find the above mentioned relations (9), (10) by hand, and one has to turn to the software for symbolic manipulations. We discuss some aspects of symbolic computations in the next section.

\section{Hirota-Kimura bases}

For our method, the notion of a Hirota-Kimura basis, introduced and studied in some detail in [12], is relevant. We recall here the main facts, following that reference.

For a given birational map $f: \mathbb{R}^{n} \rightarrow \mathbb{R}^{n}$, a set of functions $\Phi=\left(\varphi_{1}, \ldots, \varphi_{m}\right)$, linearly independent over $\mathbb{R}$, is called a Hirota-Kimura basis (HK-basis), if for every $x \in \mathbb{R}^{n}$ there exists a non-vanishing vector $c=\left(c_{1}, \ldots, c_{m}\right)^{\mathrm{T}}$ such that

$$
c_{1} \varphi_{1}\left(f^{i}(x)\right)+\ldots+c_{m} \varphi_{m}\left(f^{i}(x)\right)=0 \text { for all } i \in \mathbb{Z} \text {. }
$$

For a given $x \in \mathbb{R}^{n}$, the vector space consisting of all $c \in \mathbb{R}^{m}$ with this property, say $K_{\Phi}(x)$, is called the null-space of the basis $\Phi$ at the point $x$.

The notion of HK-bases is closely related to the notion of integrals, even if they cannot be immediately translated into one another. For instance, if $K_{\Phi}(x)=\operatorname{span}\left(c_{1}, \ldots, c_{M}\right)^{\mathrm{T}}$, so that $\operatorname{dim} K_{\Phi}(x)=1$, then the quotients $c_{i}: c_{j}$ are integrals of motion (or, in other words, $\left[c_{1}: \ldots: c_{m}\right] \in \mathbb{C P}^{m-1}$ is an integral of motion).

For a given set of functions $\Phi=\left(\varphi_{1}, \ldots, \varphi_{m}\right)$ and for any interval $[j, k] \subset \mathbb{Z}$ we denote

$$
X_{[j, k]}(x)=\left(\begin{array}{ccc}
\varphi_{1}\left(f^{j}(x)\right) & . . & \varphi_{m}\left(f^{j}(x)\right) \\
\varphi_{1}\left(f^{j+1}(x)\right) & . . & \varphi_{m}\left(f^{j+1}(x)\right) \\
\ldots & & \ldots \\
\varphi_{1}\left(f^{k}(x)\right) & . . & \varphi_{m}\left(f^{k}(x)\right)
\end{array}\right) .
$$

In particular, $X_{(-\infty, \infty)}(x)$ will denote the double infinite matrix of the type (11). Obviously,

$$
\operatorname{ker} X_{(-\infty, \infty)}(x)=K_{\Phi}(x)
$$


Thus, $\Phi$ is a Hirota-Kimura basis for $f$ if and only if $\operatorname{dim} \operatorname{ker} X_{(-\infty, \infty)}(x) \geq 1$. Our algorithm for detecting this situation is based on the following result formulated in [12].

Theorem 5. Let

$$
\operatorname{dim} \operatorname{ker} X_{[0, s-1]}(x)=m-s \text { for } 1 \leq s \leq m-1 \text {, }
$$

and

$$
\operatorname{dim} \operatorname{ker} X_{[0, m-1]}(x)=1
$$

hold for all $x \in \mathbb{R}^{n}$. Then for any $x \in \mathbb{R}^{n}$ there holds:

$$
K_{\Phi}(x)=\operatorname{ker} X_{[0, m-1]}(x),
$$

and, in particular,

$$
\operatorname{dim} K_{\Phi}(x)=1 \text {. }
$$

Let us stress that, while in general (i.e., for general maps $f$, general function sets $\Phi$ and for generic $x \in \mathbb{R}^{n}$ ) relations (12) are satisfied, one will generally find that the $m \times$ $m$ matrix $X_{[0, m-1]}(x)$ is non-degenerate, so that dim $\operatorname{ker} X_{[0, m-1]}(x)=\operatorname{dim} K_{\Phi}(x)=0$. Finding (a candidate for) a HK-basis $\Phi$ with $\operatorname{dim} \operatorname{ker} X_{[0, m-1]}(x)=\operatorname{dim} K_{\Phi}(x)=1$ is a highly non-trivial task.

The following numerical algorithm can be used for checking that a given system of functions $\Phi=\left(\varphi_{1}, \ldots, \varphi_{m}\right)$ is a HK-basis for a given birational map $f$.

(N) For several randomly chosen initial points $x \in \mathbb{Q}^{n}$, compute $\operatorname{ker} X_{[0, m-1]}(x)$, using exact rational arithmetic. If for every $x$ condition $(13)$ is satisfied, then, for all practical considerations, $\Phi$ is a HK-basis for $f$, with $\operatorname{dim} K_{\Phi}(x)=1$.

In what follows, we will call the results obtained in this way Facts. As such, they cannot be ascribed the status of mathematical theorems, but with an additional effort they can be given a rigorous proof (sometimes a human proof, and sometimes a computer assisted one). We have provided details for such proofs in [12] in the situation which was much more demanding than the ones described in the present paper. Therefore, we are pretty sure that all our Facts can be turned into mathematical theorems after a sufficient effort. However, this would not be strictly necessary for our purposes, because we use these Facts to formulate the Ansätze for solutions, which are then rigorously proved by different methods.

The relevance of the notion of HK-bases for our present goals is easy to understand. Indeed, existence of an algebraic relation like (9) or (10) for iterates of a birational map $f$ can be phrased as existence of a certain HK-basis. For instance, formula (4) for some component $x_{i}$ of the vector $x$ is equivalent to saying that

$$
\Phi=\left(x_{i}^{2} \tilde{x}_{i}^{2}, x_{i} \tilde{x}_{i}\left(x_{i}+\tilde{x}_{i}\right), x_{i}^{2}+\tilde{x}_{i}^{2}, x_{i} \tilde{x}_{i}, x_{i}+\widetilde{x}_{i}, 1\right),
$$

is a HK basis for $f$ with $K_{\Phi}(x)$ spanned by $\left(\alpha_{0}, \ldots, \alpha_{5}\right)$. By the way, formula (3) can be interpreted as the claim that

$$
\Phi=\left(\dot{x}^{2}, x^{4}, x^{3}, x^{2}, x, 1\right)
$$

is a HK-basis with $\operatorname{dim} K_{\Phi}(x)=1$ for the phase flow of the corresponding differential equation. Thus, actually HK-bases appeared in a disguised form in the continuous time theory long ago.

In the remaining part of the paper we will apply the method described in the last two sections to finding explicit solutions, given in terms of elliptic functions, of two birational 
maps discretizing two classical integrable systems, namely the periodic Volterra chains with $N=3$ and $N=4$.

\section{ELLIPTIC SOLUTIONS OF THE INFINITE VOLTERRA CHAiN}

The infinite Volterra chain (VC) is a well-known completely integrable system [7, 11] governed by the equations of motion

$$
\dot{x}_{n}=x_{n}\left(x_{n+1}-x_{n-1}\right), \quad n \in \mathbb{Z} \text {. }
$$

This system admits two different families of solutions which are expressed in terms of elliptic functions.

The first family of elliptic solutions [16] is given by:

$$
\begin{aligned}
x_{n}(t) & =\zeta(t+n v)-\zeta(t+(n-1) v)+\zeta(v)-\zeta(2 v) \\
& =\frac{\sigma(t+(n+1) v) \sigma(t+(n-2) v)}{\sigma(t+n v) \sigma(t+(n-1) v) \sigma(2 v)}
\end{aligned}
$$

where $v$ is an arbitrary complex number. The equivalence of the two representations (15) and (16) is either easily checked by looking at poles and zeroes of the both elliptic functions, or just by using formula

$$
\zeta(a)+\zeta(b)+\zeta(c)-\zeta(a+b+c)=\frac{\sigma(a+b) \sigma(b+c) \sigma(c+a)}{\sigma(a) \sigma(b) \sigma(c) \sigma(a+b+c)}
$$

The check that (15) and (16) are indeed a solution of VC is now elementary. Take the logarithmic derivative of (16) and then use (15) with shifted indices:

$$
\begin{aligned}
\frac{\dot{x}_{n}}{x_{n}} & =\zeta(t+(n+1) v)+\zeta(t+(n-2) v)-\zeta(t+n v)-\zeta(t+(n-1) v) \\
& =x_{n+1}-x_{n-1} .
\end{aligned}
$$

The second family of elliptic solutions [10, 15] is given by:

$$
\begin{aligned}
x_{2 n-1}(t) & =\zeta\left(t+n v_{1}+(n-1) v_{2}\right)-\zeta\left(t+(n-1)\left(v_{1}+v_{2}\right)\right)+\zeta\left(v_{2}\right)-\zeta\left(v_{1}+v_{2}\right) \\
& =\frac{\sigma\left(t+n\left(v_{1}+v_{2}\right)\right) \sigma\left(t+(n-1) v_{1}+(n-2) v_{2}\right) \sigma\left(v_{1}\right)}{\sigma\left(t+n v_{1}+(n-1) v_{2}\right) \sigma\left(t+(n-1)\left(v_{1}+v_{2}\right)\right) \sigma\left(v_{2}\right) \sigma\left(v_{1}+v_{2}\right)} \\
x_{2 n}(t) & =\zeta\left(t+n\left(v_{1}+v_{2}\right)\right)-\zeta\left(t+n v_{1}+(n-1) v_{2}\right)+\zeta\left(v_{1}\right)-\zeta\left(v_{1}+v_{2}\right) \\
& =\frac{\sigma\left(t+(n+1) v_{1}+n v_{2}\right) \sigma\left(t+(n-1)\left(v_{1}+v_{2}\right)\right) \sigma\left(v_{2}\right)}{\sigma\left(t+n\left(v_{1}+v_{2}\right)\right) \sigma\left(t+n v_{1}+(n-1) v_{2}\right) \sigma\left(v_{1}\right) \sigma\left(v_{1}+v_{2}\right)}
\end{aligned}
$$


and reduces to the first one if $v_{1}=v_{2}=v$. Again, the verification of these solutions is straightforward:

$$
\begin{aligned}
\frac{\dot{x}_{2 n-1}}{x_{2 n-1}}= & \zeta\left(t+n\left(v_{1}+v_{2}\right)\right)+\zeta\left(t+(n-1) v_{1}+(n-2) v_{2}\right) \\
& -\zeta\left(t+n v_{1}+(n-1) v_{2}\right)-\zeta\left(t+(n-1)\left(v_{1}+v_{2}\right)\right) \\
= & x_{2 n}-x_{2 n-2} \\
\frac{\dot{x}_{2 n}}{x_{2 n}}= & \left.\zeta\left(t+(n+1) v_{1}+n v_{2}\right)\right)+\zeta\left(t+(n-1)\left(v_{1}+v_{2}\right)\right) \\
& -\zeta\left(t+n\left(v_{1}+v_{2}\right)\right)-\zeta\left(t+n v_{1}+(n-1) v_{2}\right) \\
= & x_{2 n+1}-x_{2 n-1} .
\end{aligned}
$$

The first family of solutions admits an $N$-periodic reduction $(n \in \mathbb{Z} / N \mathbb{Z})$, if $N v \equiv 0$ modulo the period lattice. The second family admits a $(2 N)$-periodic reduction, if $N\left(v_{1}+\right.$ $\left.v_{2}\right) \equiv 0$ modulo the period lattice. We will show that for the periodic VC with $N=3$ and $N=4$, these elliptic solutions are indeed general solutions.

\section{General SOlUtions OF THE PERIODIC VOlterRa CHAin WITH $N=3$}

The periodic reduction of the Volterra chain with $N=3\left(\mathrm{VC}_{3}\right)$ reads:

$$
\left\{\begin{array}{l}
\dot{x}_{1}=x_{1}\left(x_{2}-x_{3}\right), \\
\dot{x}_{2}=x_{2}\left(x_{3}-x_{1}\right), \\
\dot{x}_{3}=x_{3}\left(x_{1}-x_{2}\right) .
\end{array}\right.
$$

This integrable system possesses two functionally independent integrals of motion:

$$
H_{1}=x_{1}+x_{2}+x_{3}, \quad H_{2}=x_{1} x_{2} x_{3} .
$$

We now prove that formulas (15) or (16) provide indeed the general solution of $\mathrm{VC}_{3}$.

Theorem 6. The general solution of $V C_{3}$ is given by formulas (15) or (16) with $v$ being a one third of a period, i.e., $3 v \equiv 0$ modulo the period lattice. Explicitly, in terms of $\zeta$-functions,

$$
\begin{aligned}
& x_{1}(t)=\zeta(t+v)-\zeta(t)+\zeta(v)-\zeta(2 v), \\
& x_{2}(t)=\zeta(t+2 v)-\zeta(t+v)+\zeta(v)-\zeta(2 v), \\
& x_{3}(t)=\zeta(t+3 v)-\zeta(t+2 v)+\zeta(v)-\zeta(2 v),
\end{aligned}
$$

or, in terms of $\sigma$-functions,

$$
\begin{aligned}
& x_{1}(t)=\frac{\sigma(t-v) \sigma(t+2 v)}{\sigma(t) \sigma(t+v) \sigma(2 v)}, \\
& x_{2}(t)=\frac{\sigma(t) \sigma(t+3 v)}{\sigma(t+v) \sigma(t+2 v) \sigma(2 v)}, \\
& x_{3}(t)=\frac{\sigma(t+v) \sigma(t+4 v)}{\sigma(t+2 v) \sigma(t+3 v) \sigma(2 v)} .
\end{aligned}
$$

Proof. Eliminating $x_{j}, x_{k}$ from equations of motion (22) for $x_{i}$ with the help of integrals of motion (23), one arrives at

$$
\dot{x}_{i}^{2}=x_{i}^{2}\left(x_{i}-H_{1}\right)^{2}-4 H_{2} x_{i}
$$


We can now apply Theorem 1 to the differential equation (27), which shows that the general solution is given by elliptic functions. The quartic polynomial in (27) has the coefficients

$$
\alpha_{0}=1, \quad \alpha_{1}=-\frac{1}{2} H_{1}, \quad \alpha_{2}=\frac{1}{6} H_{1}^{2}, \quad \alpha_{3}=-H_{2}, \quad \alpha_{4}=0 .
$$

Thus, we find that each of the coordinates $x_{i}$ is a time shift of

$$
x(t)=\zeta(t+v)-\zeta(t)-\zeta(v)+\frac{1}{2} H_{1}
$$

where the zeta-functions correspond to the Weierstrass invariants

$$
g_{2}=-2 H_{1} H_{2}+\frac{1}{12} H_{1}^{4}, \quad g_{3}=-H_{2}^{2}+\frac{1}{6} H_{1}^{3} H_{2}-\frac{1}{216} H_{1}^{6},
$$

and the shift $v$ is determined from

$$
\wp(v)=\frac{1}{12} H_{1}^{2}, \quad \wp^{\prime}(v)=-H_{2} .
$$

From (28), (29) we find:

$$
H_{1} H_{2}=\frac{1}{24} H_{1}^{4}-\frac{1}{2} g_{2}=6 \wp^{2}(v)-\frac{1}{2} g_{2}=\wp^{\prime \prime}(v)
$$

which implies

$$
12 \wp(v)\left(\wp^{\prime}(v)\right)^{2}=\left(\wp^{\prime \prime}(v)\right)^{2}
$$

This has to be compared with the duplication formula for the $\wp$-function,

$$
\wp(2 v)=\frac{1}{4}\left(\frac{\wp^{\prime \prime}(v)}{\wp^{\prime}(v)}\right)^{2}-2 \wp(v) .
$$

As a result, we find $\wp(2 v)=\wp(v)$, so that $2 v \equiv-v$, or $3 v \equiv 0$. From the above formulas there follows:

$$
H_{1}=-\frac{\wp^{\prime \prime}(v)}{\wp^{\prime}(v)}=4 \zeta(v)-2 \zeta(2 v) .
$$

Finally, each of the coordinates $x_{i}$ is a time shift of

$$
x(t)=\zeta(t+v)-\zeta(t)-\zeta(v)+\frac{1}{2} H_{1}=\zeta(t+v)-\zeta(t)+\zeta(v)-\zeta(2 v),
$$

We may eliminate any $x_{k}$ between equations (23), getting $x_{i} x_{j}\left(x_{i}+x_{j}\right)-H_{1} x_{i} x_{j}+H_{2}=0$. Hence, any pair of functions $\left(x_{i}, x_{j}\right)$ satisfies a polynomial relation of degree 3 , which implies that any two functions $x_{i}$ and $x_{j}$ must have one common pole. Therefore, solutions of $\mathrm{VC}_{3}$ must be as in (24)-(26).

\section{General solution of HK-Discretization of Volterra CHAin With $N=3$}

We study here the discretization of the Volterra chain produced by so-called HirotaKimura (HK) scheme. This scheme seems to be introduced in the geometric integration literature by W. Kahan in the unpublished notes [8]. Kahan's discretization is applicable to any system of ordinary differential equations with a quadratic vector field, and automatically produces birational maps. Probably unaware of the work by Kahan, this scheme was applied to integrable systems, namely to the Euler top and to the Lagrange top, by R. Hirota and K. Kimura [5,9]. Later on, the authors of the present paper devoted 
a series of papers, [6, 12--14], to integrability properties of this discretization technique, providing a long list of integrable discretizations of systems belonging to the realm of classical mechanics, including Volterra chains with $N=3$ and $N=4$. In the context of integrable systems, Kahan's discretization has been called HK-discretization. This line of research was continued in [2,3].

The existence of two independent conserved quantities and of an invariant measure form of the HK-discretization of $\mathrm{VC}_{3}\left(\mathrm{dVC}_{3}\right)$ has been established in [13]. The discrete equations of motion read

$$
\left\{\begin{array}{l}
\widetilde{x}_{1}-x_{1}=\varepsilon x_{1}\left(\widetilde{x}_{2}-\widetilde{x}_{3}\right)+\varepsilon \widetilde{x}_{1}\left(x_{2}-x_{3}\right), \\
\widetilde{x}_{2}-x_{2}=\varepsilon x_{2}\left(\widetilde{x}_{3}-\widetilde{x}_{1}\right)+\varepsilon \widetilde{x}_{2}\left(x_{3}-x_{1}\right), \\
\widetilde{x}_{3}-x_{3}=\varepsilon x_{3}\left(\widetilde{x}_{1}-\widetilde{x}_{2}\right)+\varepsilon \widetilde{x}_{3}\left(x_{1}-x_{2}\right) .
\end{array}\right.
$$

The birational map generated by discrete equations of motion $\mathrm{dVC}_{3}$, possess the conserved quantities:

$$
H_{1}=x_{1}+x_{2}+x_{3}, \quad H_{2}(\varepsilon)=\frac{x_{1} x_{2} x_{3}}{1-\varepsilon^{2}\left(x_{1}^{2}+x_{2}^{2}+x_{3}^{2}-2 x_{1} x_{2}-2 x_{2} x_{3}-2 x_{3} x_{1}\right)} .
$$

To construct the general solution of $\mathrm{dVC}_{3}$ in terms of elliptic functions, we will follow the procedure described in section 2. Recall that a Fact is an experimental result obtained by a multiple run of Algorithm (N) with random initial data, which can be, in principle, given a rigorous (maybe, computer assisted) proof.

Fact 1. Along any orbit $\mathcal{O}(x)=\left\{f^{n}(x)\right\}_{n \in \mathbb{Z}}$ of the map $f: x \mapsto \tilde{x}$, the pairs $\left(x_{i}, \widetilde{x}_{i}\right)$ for all $i=1,2,3$ lie on a symmetric biquadratic curve, as in equation (4).

According to Theorem 2, we interpret Fact 1 as follows. The points of any orbit $\mathcal{O}(x)$ can be parametrized by the "phase" $t$ on an elliptic curve $\mathbb{C} / \Lambda$, where $\Lambda$ is the period lattice, so that the $f^{n}(x)$ corresponds to $t=n \delta+t_{0}$ with some $\delta \in \mathbb{C}$. Thus, the action of the map $f: x \mapsto \widetilde{x}$ is represented by a shift $t \mapsto t+\delta$ on the elliptic curve $\mathbb{C} / \Lambda$. Moreover, each component $x_{i}$ as a function of $t$ is an elliptic function of order 2. Furthermore, the biquadratic curve $P\left(x_{i}, \widetilde{x}_{i}\right)$ is one and the same for all three components $x_{1}, x_{2}, x_{3}$. Therefore all three components are obtained by shifting the argument of one and the same function. For symmetry reasons, we may assume that

$$
x_{2}(t)=x_{1}(t+v), \quad x_{3}(t)=x_{2}(t+v), \quad 3 v \equiv 0 \quad(\bmod \Lambda) .
$$

Fact 2. Along any orbit $\mathcal{O}(x)$, for each $i, j=1,2,3$, the pairs $\left(x_{i}, x_{j}\right)$ lie on a symmetric biquadratic curve of total degree 3 ,

$$
Q_{i j}\left(x_{i}, x_{j}\right)=\alpha_{1} x_{i} x_{j}\left(x_{i}+x_{j}\right)+\alpha_{2}\left(x_{i}^{2}+x_{j}^{2}\right)+\alpha_{3} x_{i} x_{j}+\alpha_{4}\left(x_{i}+x_{j}\right)+\alpha_{5}=0 .
$$

As a consequence, according to Theorem 4, every pair of functions $x_{i}$ and $x_{j}$ has one common pole. We may assume that the denominators of the functions $x_{1}, x_{2}, x_{3}$ are $\sigma(t) \sigma(t+v), \sigma(t+v) \sigma(t+2 v), \sigma(t+2 v) \sigma(t+3 v)$, respectively, just as in the solution of $\mathrm{VC}_{3}$, see (24)-26.

Fact 3. Along any orbit $\mathcal{O}(x)$, for each $i, j=1,2,3$, the pairs $\left(x_{i}, 1 / \tilde{x}_{j}\right)$ lie on a biquadratic curve of total degree 3. 
As a consequence, the functions $x_{i}, 1 / \widetilde{x}_{j}$ have a common pole. Therefore the two zeros of $x_{i}$ must be the $\delta$-shift and the $(-\delta)$-shift of the common pole of $x_{j}$ and $x_{k}$. We arrive at the conclusion that

$$
\begin{aligned}
& x_{1}(t)=\rho \frac{\sigma(t-v-\delta) \sigma(t+2 v+\delta)}{\sigma(t) \sigma(t+v)}, \\
& x_{2}(t)=\rho \frac{\sigma(t-\delta) \sigma(t+3 v+\delta)}{\sigma(t+v) \sigma(t+2 v)}, \\
& x_{3}(t)=\rho \frac{\sigma(t+v-\delta) \sigma(t+4 v+\delta)}{\sigma(t+2 v) \sigma(t+3 v)},
\end{aligned}
$$

where the factor $\rho$ has to be determined. The other choice of the signs of the time shifts leads to the same functions, up to a constant factor.

The last missing portion of information necessary for a complete solution, namely, the expressions for the factors $\rho$ in the above formulas, is contained in Fact 4 below. The idea to consider the expressions mentioned there comes from the following equivalent form of equations of motion (30):

$$
\frac{\widetilde{x}_{i}}{1+\varepsilon\left(\widetilde{x}_{j}-\widetilde{x}_{k}\right)}=\frac{x_{i}}{1-\varepsilon\left(x_{j}-x_{k}\right)},
$$

where $(i, j, k)$ is any cyclic permutation of $(1,2,3)$.

Fact 4. Along any orbit $\mathcal{O}(x)$, for each cyclic permutation $(i, j, k)$ of the indices $(1,2,3)$, the pairs

$$
\left(\frac{x_{i}}{1 \pm \varepsilon\left(x_{j}-x_{k}\right)}, \frac{\tilde{x}_{i}}{1 \pm \varepsilon\left(\widetilde{x}_{j}-\widetilde{x}_{k}\right)}\right)
$$

lie on a symmetric biquadratic curve.

As a consequence, the functions

$$
\frac{x_{i}}{1 \pm \varepsilon\left(x_{j}-x_{k}\right)}
$$

are elliptic functions of degree 2. In view of equations (35), Fact 4 yields also that the two zeros of $x_{1} /\left(1-\varepsilon\left(x_{2}-x_{3}\right)\right)$ must be $v-\delta, v$, while the two zeros of $x_{1} /\left(1+\varepsilon\left(x_{2}-x_{3}\right)\right)$ must be $v, v+\delta$. In other words, the following relations must hold true:

$$
1-\left.\varepsilon\left(x_{2}-x_{3}\right)\right|_{t=v+\delta}=0, \quad 1+\left.\varepsilon\left(x_{2}-x_{3}\right)\right|_{t=v-\delta}=0 .
$$

Upon using formulas (32)-(34) and taking into account that $3 v \equiv 0$, both requirements in (36) result in one and the same formula for the factor $\rho$, namely,

$$
\frac{1}{\varepsilon \rho}=\frac{\sigma(2 v+2 \delta) \sigma(v)}{\sigma(\delta) \sigma(v+\delta)}+\frac{\sigma(2 v) \sigma(v+2 \delta)}{\sigma(v-\delta) \sigma(\delta)} .
$$

To simplify this expression, we observe that $\sigma(2 v+\delta) \sigma(v+\delta) \sigma(v) \sigma(2 \delta)=\sigma(2 v+2 \delta) \sigma(v) \sigma(v-\delta) \sigma(\delta)+\sigma(2 v) \sigma(v+2 \delta) \sigma(v+\delta) \sigma(\delta)$, which follows from the three-term functional equation for the $\sigma$-function,

$$
\begin{gathered}
\sigma(z+a) \sigma(z-a) \sigma(b+c) \sigma(b-c)+\sigma(z+b) \sigma(z-b) \sigma(c+a) \sigma(c-a) \\
+\sigma(z+c) \sigma(z-c) \sigma(a+b) \sigma(a-b)=0
\end{gathered}
$$


with the choice

$$
z=\frac{3 v}{2}+\delta, \quad a=\frac{v}{2}+\delta, \quad b=\frac{v}{2}-\delta, \quad c=-\frac{v}{2} .
$$

Thus, we get

$$
\frac{1}{\varepsilon \rho}=\frac{\sigma(2 v+\delta) \sigma(v) \sigma(2 \delta)}{\sigma(v-\delta) \sigma^{2}(\delta)} .
$$

We arrive at the following statement, which can be now proven analytically.

Theorem 7. The general solution of $d V C_{3}$ is given by formulas (32)-(34), with $3 v \equiv 0$, where $\rho$ is given in (39). In terms of $\zeta$-functions,

$$
\begin{aligned}
& x_{1}(t)=\widetilde{\rho}(\zeta(t+v)-\zeta(t)+\zeta(v+\delta)-\zeta(2 v+\delta)), \\
& x_{2}(t)=\widetilde{\rho}(\zeta(t+2 v)-\zeta(t+v)+\zeta(v+\delta)-\zeta(2 v+\delta)), \\
& x_{3}(t)=\widetilde{\rho}(\zeta(t+3 v)-\zeta(t+2 v)+\zeta(v+\delta)-\zeta(2 v+\delta)),
\end{aligned}
$$

with

$$
\frac{1}{\varepsilon \widetilde{\rho}}=\frac{\sigma^{2}(v) \sigma(2 \delta)}{\sigma(v+\delta) \sigma(v-\delta) \sigma^{2}(\delta)}=2 \zeta(\delta)-\zeta(v+\delta)+\zeta(v-\delta) .
$$

Thus, for any initial point $x$, its iterations $f^{n}(x)$ are given by the above formulas with $t=n \delta+t_{0}$, with a suitable lattice of periods and suitable parameters $v, \delta, t_{0} \in \mathbb{C}$ (where, recall , $3 v \equiv 0$ ).

Proof. We verify that formulas (32) $-(34)$, with $3 v \equiv 0$, where $\rho$ is given in (39) provide indeed solutions of equations of motion (35). A similar computation can be done for solutions (40)-(42) written in terms of $\zeta$-functions.

More concretely we want to prove that the following discrete equation of motion is satisfied:

$$
\frac{x_{1}}{1-\varepsilon\left(x_{2}-x_{3}\right)}=\frac{\tilde{x}_{1}}{1+\varepsilon\left(\widetilde{x}_{2}-\widetilde{x}_{3}\right)} .
$$

From (37) and (33)-(34) we have:

$$
\begin{gathered}
\frac{1}{\varepsilon \rho}\left(1-\varepsilon\left(x_{2}-x_{3}\right)\right)=\frac{\sigma(2 v) \sigma(v+2 \delta)}{\sigma(v-\delta) \sigma(\delta)}+\frac{\sigma(2 v+2 \delta) \sigma(v)}{\sigma(v+\delta) \sigma(\delta)} \\
-\frac{\sigma(t-\delta) \sigma(t+3 v+\delta)}{\sigma(t+v) \sigma(t+2 v)}+\frac{\sigma(t+v-\delta) \sigma(t+4 v+\delta)}{\sigma(t+2 v) \sigma(t+3 v)} \\
=\frac{\sigma(t+v) \sigma(t+2 v) \sigma(2 v) \sigma(v+2 \delta)-\sigma(t-\delta) \sigma(t+3 v+\delta) \sigma(v-\delta) \sigma(\delta)}{\sigma(t+v) \sigma(t+2 v) \sigma(v-\delta) \sigma(\delta)} \\
+\frac{\sigma(t+2 v) \sigma(t+3 v) \sigma(2 v+2 \delta) \sigma(v)+\sigma(t+v-\delta) \sigma(t+4 v+\delta) \sigma(v+\delta) \sigma(\delta)}{\sigma(t+2 v) \sigma(t+3 v) \sigma(v+\delta) \sigma(\delta)} .
\end{gathered}
$$

Applying formula (38) twice, first with

$$
z=t+\frac{3 v}{2}, \quad a=\frac{v}{2}, \quad b=\frac{3 v}{2}+\delta, \quad c=\frac{v}{2}-\delta,
$$

and then with

$$
z=t+\frac{5 v}{2}, \quad a=\frac{v}{2}, \quad b=\frac{3 v}{2}+\delta, \quad c=\frac{v}{2}+\delta,
$$


we obtain:

$$
\begin{aligned}
\frac{1}{\varepsilon \rho}\left(1-\varepsilon\left(x_{2}-x_{3}\right)\right)= & \frac{\sigma(t+2 v-\delta) \sigma(t+v+\delta) \sigma(2 v+\delta) \sigma(v+\delta)}{\sigma(t+v) \sigma(t+2 v) \sigma(v-\delta) \sigma(\delta)} \\
& +\frac{\sigma(t+3 v+\delta) \sigma(t+2 v-\delta) \sigma(2 v+\delta) \sigma(v+\delta)}{\sigma(t+2 v) \sigma(t+3 v) \sigma(v+\delta) \sigma(\delta)} \\
= & \frac{\sigma(t+2 v-\delta) \sigma(2 v+\delta) \sigma(v+\delta)}{\sigma(t+2 v) \sigma(\delta)} \\
& \times\left(\frac{\sigma(t+v+\delta)}{\sigma(v-\delta) \sigma(t+v)}+\frac{\sigma(t+3 v+\delta)}{\sigma(v+\delta) \sigma(t+3 v)}\right) .
\end{aligned}
$$

A similar computation gives

$$
\begin{aligned}
\frac{1}{\varepsilon \rho}\left(1+\varepsilon\left(\widetilde{x}_{2}-\widetilde{x}_{3}\right)\right)= & \frac{\sigma(t+2 v+2 \delta) \sigma(2 v+\delta) \sigma(v+\delta)}{\sigma(t+2 v+\delta) \sigma(\delta)} \\
& \times\left(\frac{\sigma(t+v)}{\sigma(v+\delta) \sigma(t+v+\delta)}+\frac{\sigma(t+3 v)}{\sigma(v-\delta) \sigma(t+3 v+\delta)}\right) .
\end{aligned}
$$

Now a straightforward computation leads to

$$
\begin{aligned}
& \frac{1-\varepsilon\left(x_{2}-x_{3}\right)}{x_{1}}=\varepsilon \frac{\sigma(2 v+\delta)}{\sigma(\delta) \sigma(v-\delta)} A(t, v, \delta) C_{1}(t, v, \delta), \\
& \frac{1+\varepsilon\left(\widetilde{x}_{2}-\widetilde{x}_{3}\right)}{\widetilde{x}_{1}}=\varepsilon \frac{\sigma(2 v+\delta)}{\sigma(\delta) \sigma(v-\delta)} A(t, v, \delta) C_{2}(t, v, \delta),
\end{aligned}
$$

where

$$
A(t, v, \delta)=\frac{\sigma(t+v+\delta) \sigma(t+3 v) \sigma(v+\delta)+\sigma(t+v) \sigma(t+3 v+\delta) \sigma(v-\delta)}{\sigma(t+2 v) \sigma(t+2 v+\delta)}
$$

and

$$
\begin{aligned}
& C_{1}(t, v, \delta)=\frac{\sigma(t) \sigma(t+2 v-\delta)}{\sigma(t-v-\delta) \sigma(t+3 v)}, \\
& C_{2}(t, v, \delta)=\frac{\sigma(t+\delta) \sigma(t+2 v)}{\sigma(t-v) \sigma(t+3 v+\delta)} .
\end{aligned}
$$

The functions $C_{1}(t, v, \delta)$ and $C_{2}(t, v, \delta)$ are both constant (and equal to one another), since they are elliptic functions without zeros and poles, due to $3 v \equiv 0$. The Theorem is thus proved.

\section{General solutions of the Periodic VOlterra CHAin With $N=4$}

The periodic reduction of the Volterra chain with $N=4\left(\mathrm{VC}_{4}\right)$ reads:

$$
\left\{\begin{array}{l}
\dot{x}_{1}=x_{1}\left(x_{2}-x_{4}\right), \\
\dot{x}_{2}=x_{2}\left(x_{3}-x_{1}\right), \\
\dot{x}_{3}=x_{3}\left(x_{4}-x_{2}\right), \\
\dot{x}_{4}=x_{4}\left(x_{1}-x_{3}\right) .
\end{array}\right.
$$

This integrable system possesses three functionally independent integrals of motion:

$$
H_{1}=x_{1}+x_{2}+x_{3}+x_{4}, \quad H_{2}=x_{1} x_{3}, \quad H_{3}=x_{2} x_{4} .
$$


We now prove that formulas $(18)-(21)$ provide indeed the general solution of $\mathrm{VC}_{4}$.

Theorem 8. The general solution of $V C_{4}$ is given by formulas (18)-(21) with $2\left(v_{1}+v_{2}\right) \equiv 0$. Explicitly, in terms of $\zeta$-functions,

$$
\begin{aligned}
& x_{1}(t)=\zeta\left(t+v_{1}\right)-\zeta(t)+\zeta\left(v_{2}\right)-\zeta\left(v_{1}+v_{2}\right) \\
& x_{2}(t)=\zeta\left(t+v_{1}+v_{2}\right)-\zeta\left(t+v_{1}\right)+\zeta\left(v_{1}\right)-\zeta\left(v_{1}+v_{2}\right), \\
& x_{3}(t)=\zeta\left(t+2 v_{1}+v_{2}\right)-\zeta\left(t+v_{1}+v_{2}\right)+\zeta\left(v_{2}\right)-\zeta\left(v_{1}+v_{2}\right), \\
& x_{4}(t)=\zeta\left(t+2 v_{1}+2 v_{2}\right)-\zeta\left(t+2 v_{1}+v_{2}\right)+\zeta\left(v_{1}\right)-\zeta\left(v_{1}+v_{2}\right) .
\end{aligned}
$$

In terms of $\sigma$-functions,

$$
\begin{aligned}
& x_{1}(t)=\rho_{1} \frac{\sigma\left(t-v_{2}\right) \sigma\left(t+v_{1}+v_{2}\right)}{\sigma(t) \sigma\left(t+v_{1}\right)}, \\
& x_{2}(t)=\rho_{2} \frac{\sigma(t) \sigma\left(t+2 v_{1}+v_{2}\right)}{\sigma\left(t+v_{1}\right) \sigma\left(t+v_{1}+v_{2}\right)}, \\
& x_{3}(t)=\rho_{1} \frac{\sigma\left(t+v_{1}\right) \sigma\left(t+2 v_{1}+2 v_{2}\right)}{\sigma\left(t+v_{1}+v_{2}\right) \sigma\left(t+2 v_{1}+v_{2}\right)}, \\
& x_{4}(t)=\rho_{2} \frac{\sigma\left(t+v_{1}+v_{2}\right) \sigma\left(t+3 v_{1}+2 v_{2}\right)}{\sigma\left(t+2 v_{1}+v_{2}\right) \sigma\left(t+2 v_{1}+2 v_{2}\right)},
\end{aligned}
$$

where

$$
\rho_{1}=\frac{\sigma\left(v_{1}\right)}{\sigma\left(v_{2}\right) \sigma\left(v_{1}+v_{2}\right)}, \quad \rho_{2}=\frac{\sigma\left(v_{2}\right)}{\sigma\left(v_{1}\right) \sigma\left(v_{1}+v_{2}\right)} .
$$

Proof. One easily finds that $x_{1}, x_{3}$ satisfy the differential equation

$$
\dot{x}^{2}=\left(x^{2}-H_{1} x+H_{2}\right)^{2}-4 H_{3} x^{2},
$$

while $x_{2}, x_{4}$ satisfy a similar equation with $H_{2} \leftrightarrow H_{3}$. This is a differential equation of the form (3) with

$$
\alpha_{0}=1, \quad \alpha_{1}=-\frac{1}{2} H_{1}, \quad \alpha_{2}=\frac{1}{6}\left(H_{1}^{2}+2 H_{2}-4 H_{3}\right), \quad \alpha_{3}=-\frac{1}{2} H_{1} H_{2}, \quad \alpha_{4}=H_{2}^{2} .
$$

Theorem 1 immediately leads to solution in terms of elliptic functions. The Weierstrass invariants can be expressed as

$$
\begin{aligned}
g_{2}= & \frac{1}{12} H_{1}^{4}-\frac{2}{3} H_{1}^{2}\left(H_{2}+H_{3}\right)+\frac{4}{3}\left(H_{2}^{2}+H_{3}^{2}-H_{2} H_{3}\right), \\
g_{3}= & -\frac{1}{216} H_{1}^{6}+\frac{1}{18} H_{1}^{4}\left(H_{2}+H_{3}\right)-\frac{1}{9} H_{1}^{2}\left(2 H_{2}^{2}+2 H_{3}^{2}+H_{2} H_{3}\right) \\
& +\frac{8}{27}\left(H_{2}^{3}+H_{3}^{3}\right)-\frac{4}{9} H_{2} H_{3}\left(H_{2}+H_{3}\right) .
\end{aligned}
$$

Note that $g_{2}$ and $g_{3}$ are symmetric with respect to the interchange $H_{2} \leftrightarrow H_{3}$, which implies that all functions $x_{i}$ are elliptic functions with respect to the same period lattice.

We conclude that $x_{1}$ and $x_{3}$ are given by time shifts of the function

where

$$
x_{1,3}(t)=\zeta\left(t+v_{1}\right)-\zeta(t)-\zeta\left(v_{1}\right)+\frac{1}{2} H_{1},
$$

$$
\wp\left(v_{1}\right)=\frac{1}{12} H_{1}^{2}-\frac{1}{3} H_{2}+\frac{2}{3} H_{3}, \quad \wp^{\prime}\left(v_{1}\right)=-H_{3} H_{1} .
$$


Similarly, $x_{2}$ and $x_{4}$ are time shifts of the function

$$
x_{2,4}(t)=\zeta\left(t+v_{2}\right)-\zeta(t)-\zeta\left(v_{2}\right)+\frac{1}{2} H_{1}
$$

with

$$
\wp\left(v_{2}\right)=\frac{1}{12} H_{1}^{2}-\frac{1}{3} H_{3}+\frac{2}{3} H_{2}, \quad \wp^{\prime}\left(v_{2}\right)=-H_{2} H_{1} .
$$

With the help of the addition formula

$$
\wp\left(v_{1}\right)+\wp\left(v_{2}\right)+\wp\left(v_{1}+v_{2}\right)=\frac{1}{4}\left(\frac{\wp^{\prime}\left(v_{1}\right)-\wp^{\prime}\left(v_{2}\right)}{\wp\left(v_{1}\right)-\wp\left(v_{2}\right)}\right)^{2},
$$

we find:

which yields

$$
\wp\left(v_{1}+v_{2}\right)=\frac{1}{12} H_{1}^{2}-\frac{1}{3} H_{2}-\frac{1}{3} H_{3}
$$

$$
4\left(\wp\left(v_{1}+v_{2}\right)\right)^{3}-g_{2} \wp\left(v_{1}+v_{2}\right)-g_{3}=0 .
$$

Thus, $\wp\left(v_{1}+v_{2}\right)$ is one of the roots of the Weierstrass cubic equation $4 z^{3}-g_{2} z-g_{3}=0$, which implies that $v_{1}+v_{2}$ is a half period modulo the period lattice, $2\left(v_{1}+v_{2}\right) \equiv 0$.

\section{General SOlution OF HK-DiscretizATION OF PERIODiC VOLTERRA CHAin With}

$$
N=4
$$

The existence of three independent conserved quantities and of an invariant measure form of the $\mathrm{HK}$-discretization of $\mathrm{VC}_{4}\left(\mathrm{dVC}_{4}\right)$ has been established in [13]. The discrete equations of motion read:

$$
\left\{\begin{array}{l}
\widetilde{x}_{1}-x_{1}=\varepsilon x_{1}\left(\widetilde{x}_{2}-\widetilde{x}_{4}\right)+\varepsilon \widetilde{x}_{1}\left(x_{2}-x_{4}\right), \\
\widetilde{x}_{2}-x_{2}=\varepsilon x_{2}\left(\widetilde{x}_{3}-\widetilde{x}_{1}\right)+\varepsilon \widetilde{x}_{2}\left(x_{3}-x_{1}\right), \\
\widetilde{x}_{3}-x_{3}=\varepsilon x_{3}\left(\widetilde{x}_{4}-\widetilde{x}_{2}\right)+\varepsilon \widetilde{x}_{3}\left(x_{4}-x_{2}\right), \\
\widetilde{x}_{4}-x_{4}=\varepsilon x_{4}\left(\widetilde{x}_{1}-\widetilde{x}_{3}\right)+\varepsilon \widetilde{x}_{4}\left(x_{1}-x_{4}\right) .
\end{array}\right.
$$

The birational map generated by the discrete equations of motion $\mathrm{dVC}_{4}$, possess the conserved quantities:

$$
H_{1}=x_{1}+x_{2}+x_{3}+x_{4}, \quad H_{2}(\varepsilon)=\frac{x_{1} x_{3}}{1-\varepsilon^{2}\left(x_{2}-x_{4}\right)^{2}}, \quad H_{3}(\varepsilon)=\frac{x_{2} x_{4}}{1-\varepsilon^{2}\left(x_{1}-x_{3}\right)^{2}} .
$$

We are interested in constructing explicit elliptic solutions of $\mathrm{dVC}_{4}$. To do so we will proceed as in the case of $\mathrm{dVC}_{3}$, starting by collecting preliminary experimental results about the distribution of the points $\left(x_{i}, \widetilde{x}_{i}\right)$ and $\left(x_{i}, x_{j}\right)$ on some curves.

Fact 5. Along any orbit $\mathcal{O}(x)$, for each $i=1,2,3,4$, the pairs $\left(x_{i}, \tilde{x}_{i}\right)$ lie on a curve of bidegree $(4,4)$ without constant and linear terms. Equivalently, the pairs $\left(1 / x_{i}, 1 / \widetilde{x}_{i}\right)$ lie on a curve of bidegree $(4,4)$ and of total degree 6 . The corresponding curves coincide for $i=1,3$ and for $i=2,4$. They all are of genus 1 .

As a consequence, $x_{i}$ as functions of $t$ are elliptic functions of degree 4 . Moreover, $x_{i}$ and $\widetilde{x}_{i}$ have two common zeros.

Fact 6. Along any orbit $\mathcal{O}(x)$, the pairs $\left(x_{i}, x_{j}\right)$ lie on a curve of degree 4 if $i, j$ are of different parity, and on a curve of degree 2 if $i, j$ are of the same parity. 
As a consequence, $x_{1}$ and $x_{3}$ are time shifts of one and the same function, and the same for $x_{2}$ and $x_{4}$.

Fact 7. Along any orbit $\mathcal{O}(x)$, the pairs $\left(x_{1}+x_{3}, \widetilde{x}_{1}+\widetilde{x}_{3}\right)$ lie on a biquadratic curve, and the same holds true for the pairs $\left(x_{2}+x_{4}, \widetilde{x}_{2}+\widetilde{x}_{4}\right)$.

As a consequence, functions $x_{1}+x_{3}$ and $x_{2}+x_{4}$ are of degree 2. Thus, the time shift relating $x_{1}$ and $x_{3}$ should be a half-period, and the same for $x_{2}$ and $x_{4}$. Therefore, we assume

$$
x_{3}(t)=x_{1}\left(t+v_{1}+v_{2}\right), \quad x_{4}(t)=x_{2}\left(t+v_{1}+v_{2}\right), \quad 2\left(v_{1}+v_{2}\right) \equiv 0 .
$$

We denote the common poles of $x_{i}$ by $0,-v_{1},-\left(v_{1}+v_{2}\right),-\left(2 v_{1}+v_{2}\right)$.

With the help of all this information, we can proceed as follows. Denote the zeros of $x_{1}$ by $-a,-(a-\delta),-b,-(b-\delta)$, and the zeros of $x_{2}$ by $-c,-(c-\delta),-d,-(d-\delta)$. Thus, we can finally write down the factorized expressions for $x_{1}, x_{2}$ :

$$
\begin{aligned}
& x_{1}(t)=\rho_{1} \frac{\sigma(t+a) \sigma\left(t+a+2 v_{1}+2 v_{2}-\delta\right) \sigma(t+b) \sigma(t+b-\delta)}{\sigma(t) \sigma\left(t+v_{1}\right) \sigma\left(t+v_{1}+v_{2}\right) \sigma\left(t+2 v_{1}+v_{2}\right)}, \\
& x_{2}(t)=\rho_{2} \frac{\sigma(t+c) \sigma\left(t+c+2 v_{1}+2 v_{2}-\delta\right) \sigma(t+d) \sigma(t+d-\delta)}{\sigma\left(t+v_{1}\right) \sigma\left(t+v_{1}+v_{2}\right) \sigma\left(t+2 v_{1}+v_{2}\right) \sigma\left(t+2 v_{1}+2 v_{2}\right)}
\end{aligned}
$$

where $\rho_{1}$ and $\rho_{2}$ are two factors to be determined. This choice of the factorization is justified by the continuous limit, $\delta \approx 2 \varepsilon \rightarrow 0$, of (51)-(52) which has to be compared with (44) and (46). Such a limit tells us that

$$
\begin{gathered}
a \approx-v_{2}, \quad b \approx v_{1}+v_{2}, \quad b+a-\delta=v_{1}, \\
c \approx 0, \quad d \approx 2 v_{1}+v_{2}, \quad c+d-\delta=2 v_{1}+v_{2} .
\end{gathered}
$$

Eliminating $b$ and $d$ from the above expressions, we find:

$$
\begin{aligned}
& x_{1}(t)=\rho_{1} \frac{\sigma(t+a) \sigma\left(t-a+v_{1}+\delta\right) \sigma\left(t-a+v_{1}\right) \sigma\left(t+a+2 v_{1}+2 v_{2}-\delta\right)}{\sigma(t) \sigma\left(t+v_{1}\right) \sigma\left(t+v_{1}+v_{2}\right) \sigma\left(t+2 v_{1}+v_{2}\right)} \\
& x_{2}(t)=\rho_{2} \frac{\sigma(t+c) \sigma\left(t-c+2 v_{1}+v_{2}\right) \sigma\left(t-c+2 v_{1}+v_{2}+\delta\right) \sigma\left(t+c+2 v_{1}+2 v_{2}-\delta\right)}{\sigma\left(t+v_{1}\right) \sigma\left(t+v_{1}+v_{2}\right) \sigma\left(t+2 v_{1}+v_{2}\right) \sigma\left(t+2 v_{1}+2 v_{2}\right)} .
\end{aligned}
$$

Factorized expressions for $x_{3}, x_{4}$ follow from (50). However, it turns out to be convenient to have expressions for these variables with the same denominators as for $x_{1}, x_{2}$, respectively. This is achieved by using the quasi-periodicity of the $\sigma$-function with respect to the period $2\left(v_{1}+v_{2}\right) \equiv 0$. We get:

$$
\begin{aligned}
& x_{3}(t)=\rho_{1} \frac{\sigma\left(t-a-v_{2}+\delta\right) \sigma\left(t+a+v_{1}+v_{2}\right) \sigma\left(t+a+v_{1}+v_{2}-\delta\right) \sigma\left(t-a+2 v_{1}+v_{2}\right)}{\sigma(t) \sigma\left(t+v_{1}\right) \sigma\left(t+v_{1}+v_{2}\right) \sigma\left(t+2 v_{1}+v_{2}\right)}, \\
& x_{4}(t)=\rho_{2} \frac{\sigma\left(t-c+v_{1}+\delta\right) \sigma\left(t+c+v_{1}+v_{2}\right) \sigma\left(t+c+v_{1}+v_{2}-\delta\right) \sigma\left(t-c+3 v_{1}+2 v_{2}\right)}{\sigma\left(t+v_{1}\right) \sigma\left(t+v_{1}+v_{2}\right) \sigma\left(t+2 v_{1}+v_{2}\right) \sigma\left(t+2 v_{1}+2 v_{2}\right)} .
\end{aligned}
$$


Next, we have to find the remaining unknowns $a$ and $c$, as well as $\rho_{1}$ and $\rho_{2}$. The idea to consider expressions from the crucial Fact 8 formulated below, comes from the observation that equations of motion (49) can be equivalently re-written as

$$
\begin{aligned}
\frac{\widetilde{x}_{1}}{1+\varepsilon\left(\widetilde{x}_{2}-\widetilde{x}_{4}\right)} & =\frac{x_{1}}{1-\varepsilon\left(x_{2}-x_{4}\right)}, \\
\frac{\widetilde{x}_{2}}{1+\varepsilon\left(\widetilde{x}_{3}-\widetilde{x}_{1}\right)} & =\frac{x_{2}}{1-\varepsilon\left(x_{3}-x_{1}\right)}, \\
\frac{\widetilde{x}_{3}}{1+\varepsilon\left(\widetilde{x}_{4}-\widetilde{x}_{2}\right)} & =\frac{x_{3}}{1-\varepsilon\left(x_{4}-x_{2}\right)}, \\
\frac{\widetilde{x}_{4}}{1+\varepsilon\left(\widetilde{x}_{1}-\widetilde{x}_{3}\right)} & =\frac{x_{2}}{1-\varepsilon\left(x_{1}-x_{3}\right)} .
\end{aligned}
$$

Fact 8. Along any orbit $\mathcal{O}(x)$, for each $i=1,2,3,4$, the pairs

$$
\left(\frac{x_{i}}{1 \pm \varepsilon\left(x_{j}-x_{k}\right)}, \frac{\tilde{x}_{i}}{1 \pm \varepsilon\left(\widetilde{x}_{j}-\widetilde{x}_{k}\right)}\right),
$$

where $j=i+1(\bmod 4), k=i-1(\bmod 4)$, lie on a symmetric biquadratic curve.

As a consequence, the combinations

$$
\frac{x_{i}}{1 \pm \varepsilon\left(x_{j}-x_{k}\right)}
$$

are elliptic functions of degree 2. The structure of zeros of these functions can be inferred from equations of motion (53)-(56). For instance, from (53) we see that the two zeros of $x_{1} /\left(1-\varepsilon\left(x_{2}-x_{4}\right)\right)$ are $-a,-b$, while the two zeros of $x_{1} /\left(1+\varepsilon\left(x_{2}-x_{4}\right)\right)$ are $-(a-$ $\delta),-(b-\delta)$. Therefore,

$$
\begin{array}{cl}
1-\left.\varepsilon\left(x_{2}-x_{4}\right)\right|_{t=-a+\delta}=0, & 1-\left.\varepsilon\left(x_{2}-x_{4}\right)\right|_{t=-b+\delta}=0, \\
1+\left.\varepsilon\left(x_{2}-x_{4}\right)\right|_{t=-a}=0, & 1+\left.\varepsilon\left(x_{2}-x_{4}\right)\right|_{t=-b}=0 .
\end{array}
$$

Similarly, from (54) there follows that the two zeros of $x_{2} /\left(1-\varepsilon\left(x_{3}-x_{1}\right)\right)$ are $-c,-d$, while the two zeros of $x_{2} /\left(1+\varepsilon\left(x_{3}-x_{1}\right)\right)$ are $-(c-\delta),-(d-\delta)$, so that

$$
\begin{array}{cl}
1-\left.\varepsilon\left(x_{3}-x_{1}\right)\right|_{t=-c+\delta}=0, & 1-\left.\varepsilon\left(x_{3}-x_{1}\right)\right|_{t=-d+\delta}=0, \\
1+\left.\varepsilon\left(x_{3}-x_{1}\right)\right|_{t=-c}=0, & 1+\left.\varepsilon\left(x_{3}-x_{1}\right)\right|_{t=-d}=0 .
\end{array}
$$

From (55) we deduce that the two zeros of $x_{3} /\left(1-\varepsilon\left(x_{4}-x_{2}\right)\right)$ are $-a+v_{1}+v_{2},-b+v_{1}+$ $v_{2}$, while the two zeros of $x_{3} /\left(1+\varepsilon\left(x_{4}-x_{2}\right)\right)$ are $-(a-\delta)+v_{1}+v_{2},-(b-\delta)+v_{1}+v_{2}$, so that

$$
\begin{array}{cl}
1-\left.\varepsilon\left(x_{4}-x_{2}\right)\right|_{t=-a+\delta+v_{1}+v_{2}}=0, & 1-\left.\varepsilon\left(x_{4}-x_{2}\right)\right|_{t=-b+\delta+v_{1}+v_{2}}=0, \\
1+\left.\varepsilon\left(x_{4}-x_{2}\right)\right|_{t=-a+v_{1}+v_{2}}=0, & 1+\left.\varepsilon\left(x_{4}-x_{2}\right)\right|_{t=-b+v_{1}+v_{2}}=0 .
\end{array}
$$

Finally, from (56) we conclude that the two zeros of $x_{4} /\left(1-\varepsilon\left(x_{1}-x_{3}\right)\right)$ are $-c+v_{1}+$ $v_{2},-d+v_{1}+v_{2}$, while the two zeros of $x_{4} /\left(1+\varepsilon\left(x_{1}-x_{3}\right)\right)$ are $-(c-\delta)+v_{1}+v_{2},-(d-$ $\delta)+v_{1}+v_{2}$, so that

$$
1-\left.\varepsilon\left(x_{1}-x_{3}\right)\right|_{t=-c+\delta+v_{1}+v_{2}}=0, \quad 1-\left.\varepsilon\left(x_{1}-x_{3}\right)\right|_{t=-d+\delta+v_{1}+v_{2}}=0,
$$




$$
1+\left.\varepsilon\left(x_{1}-x_{3}\right)\right|_{t=-c+v_{1}+v_{2}}=0, \quad 1+\left.\varepsilon\left(x_{1}-x_{3}\right)\right|_{t=-d+v_{1}+v_{2}}=0 .
$$

Let us first concentrate on (59) $-(60)$ and (63) $-(64)$. They result in eight conditions for $a$, $c$ and $\rho_{1}$. We show that actually almost all these conditions are equivalent, so that we are actually left with one condition for $c$ and one expression for $\rho_{1}$ through $c$ and $a$. For this aim, we first apply the tree-term formula (38) with

$$
z=t+\frac{v_{1}}{2}, \quad a=a-\frac{v_{1}}{2}, \quad b=\frac{v_{1}}{2}+v_{2}+a-\delta, \quad c=t+\frac{3 v_{1}}{2}+v_{2} .
$$

to obtain the following expression:

$$
\begin{aligned}
\frac{1}{\rho_{1}}\left(x_{1}-x_{3}\right) & =\frac{\sigma(t+a) \sigma\left(t-a+v_{1}\right) \sigma\left(t-a+v_{1}+\delta\right) \sigma\left(t+a+2 v_{1}+2 v_{2}-\delta\right)}{\sigma(t) \sigma\left(t+v_{1}\right) \sigma\left(t+v_{1}+v_{2}\right) \sigma\left(t+2 v_{1}+v_{2}\right)} \\
-\frac{\sigma\left(t-a-v_{2}+\delta\right) \sigma\left(t+a+v_{1}+v_{2}-\delta\right) \sigma\left(t+a+v_{1}+v_{2}\right) \sigma\left(t-a+2 v_{1}+v_{2}\right)}{\sigma(t) \sigma\left(t+v_{1}\right) \sigma\left(t+v_{1}+v_{2}\right) \sigma\left(t+2 v_{1}+v_{2}\right)} & \\
& =-\frac{\sigma\left(2 t+2 v_{1}+v_{2}\right) \sigma\left(v_{1}+v_{2}\right) \sigma\left(v_{1}+v_{2}-\delta\right) \sigma\left(2 a+v_{2}-\delta\right)}{\sigma(t) \sigma\left(t+v_{1}\right) \sigma\left(t+v_{1}+v_{2}\right) \sigma\left(t+2 v_{1}+v_{2}\right)} .
\end{aligned}
$$

This function changes its sign by the shift $t \mapsto t+v_{1}+v_{2}$. Therefore conditions (63)(64) are equivalent to (59)-(60). Furthermore, the above function changes it sign by $t \mapsto$ $-t-2 v_{1}-v_{2}$, therefore conditions (59) and (60) are equivalent. Thus, we can consider the first conditions in each of (59)-(60) only. They result in two different values for $\rho_{1}$ :

$$
\begin{aligned}
\varepsilon \rho_{1} & =-\frac{\sigma(c) \sigma\left(-c+v_{1}\right) \sigma\left(-c+v_{1}+v_{2}\right) \sigma\left(-c+2 v_{1}+v_{2}\right)}{\sigma\left(-2 c+2 v_{1}+v_{2}\right) \sigma\left(v_{1}+v_{2}\right) \sigma\left(v_{1}+v_{2}-\delta\right) \sigma\left(2 a+v_{2}-\delta\right)} \\
& =-\frac{\sigma(-c+\delta) \sigma\left(-c+v_{1}+\delta\right) \sigma\left(-c+v_{1}+v_{2}+\delta\right) \sigma\left(-c+2 v_{1}+v_{2}+\delta\right)}{\sigma\left(-2 c+2 v_{1}+v_{2}+2 \delta\right) \sigma\left(v_{1}+v_{2}\right) \sigma\left(v_{1}+v_{2}-\delta\right) \sigma\left(2 a+v_{2}-\delta\right)}
\end{aligned}
$$

which are equivalent if and only if the following condition holds:

$$
\frac{\sigma(c) \sigma\left(v_{1}-c\right) \sigma\left(v_{1}+v_{2}-c\right) \sigma\left(2 v_{1}+v_{2}-c\right) \sigma\left(2 v_{1}+v_{2}+2 \delta-2 c\right)}{\sigma(\delta-c) \sigma\left(v_{1}+\delta-c\right) \sigma\left(v_{1}+v_{2}+\delta-c\right) \sigma\left(2 v_{1}+v_{2}+\delta-c\right) \sigma\left(2 v_{1}+v_{2}-2 c\right)}=1 .
$$

Equation (65) determines $c$.

A similar computation can be performed for the function $\left(x_{2}-x_{4}\right) / \rho_{2}$. It turns first out that conditions (61)-(62) are equivalent to (57)-(58). Then we find that (57) is indeed equivalent to (58). We get two different values for $\rho_{2}$ :

$$
\begin{aligned}
\varepsilon \rho_{2} & =\frac{\sigma\left(a+v_{2}\right) \sigma(a) \sigma\left(-a+v_{1}\right) \sigma\left(-a+v_{1}+v_{2}\right)}{\sigma\left(-2 a+v_{1}\right) \sigma\left(v_{1}+v_{2}\right) \sigma\left(v_{1}+v_{2}-\delta\right) \sigma\left(2 c-v_{1}-\delta\right)} \\
& =-\frac{\sigma\left(-a-v_{2}+\delta\right) \sigma(-a+\delta) \sigma\left(-a+v_{1}+\delta\right) \sigma\left(-a+v_{1}+v_{2}+\delta\right)}{\sigma\left(-2 a+v_{1}+2 \delta\right) \sigma\left(v_{1}+v_{2}\right) \sigma\left(v_{1}+v_{2}-\delta\right) \sigma\left(2 c-v_{1}-\delta\right)},
\end{aligned}
$$

which are equivalent if and only if the following condition holds:

$$
\frac{\sigma(a) \sigma\left(a+v_{2}\right) \sigma\left(a-v_{1}\right) \sigma\left(-a+v_{1}+v_{2}\right) \sigma\left(-2 a+v_{1}+2 \delta\right)}{\sigma\left(\delta-a-v_{2}\right) \sigma(-a+\delta) \sigma\left(-a+v_{1}+\delta\right) \sigma\left(-a+v_{1}+v_{2}+\delta\right) \sigma\left(-2 a+v_{1}\right)}=1
$$

Equation (66) determines $a$. It is easy to see that equation (65) for $c$ and equation (66) for $a+v_{2}$ are obtained from one another by the flip $v_{1} \leftrightarrow v_{2}$ (as they should).

We are now ready to prove the desired result. 
Theorem 9. The general solution of $d V C_{4}$ is given by

$$
\begin{aligned}
& x_{1}(t)=\rho_{1} \frac{\sigma(t+a) \sigma\left(t-a+v_{1}+\delta\right) \sigma\left(t-a+v_{1}\right) \sigma\left(t+a+2 v_{1}+2 v_{2}-\delta\right)}{\sigma(t) \sigma\left(t+v_{1}\right) \sigma\left(t+v_{1}+v_{2}\right) \sigma\left(t+2 v_{1}+v_{2}\right)}, \\
& x_{2}(t)=\rho_{2} \frac{\sigma(t+c) \sigma\left(t-c+2 v_{1}+v_{2}\right) \sigma\left(t-c+2 v_{1}+v_{2}+\delta\right) \sigma\left(t+c+2 v_{1}+2 v_{2}-\delta\right)}{\sigma\left(t+v_{1}\right) \sigma\left(t+v_{1}+v_{2}\right) \sigma\left(t+2 v_{1}+v_{2}\right) \sigma\left(t+2 v_{1}+2 v_{2}\right)}, \\
& x_{3}(t)=\rho_{1} \frac{\sigma\left(t-a-v_{2}+\delta\right) \sigma\left(t+a+v_{1}+v_{2}\right) \sigma\left(t+a+v_{1}+v_{2}-\delta\right) \sigma\left(t-a+2 v_{1}+v_{2}\right)}{\sigma(t) \sigma\left(t+v_{1}\right) \sigma\left(t+v_{1}+v_{2}\right) \sigma\left(t+2 v_{1}+v_{2}\right)} \\
& x_{4}(t)=\rho_{2} \frac{\sigma\left(t-c+v_{1}+\delta\right) \sigma\left(t+c+v_{1}+v_{2}\right) \sigma\left(t+c+v_{1}+v_{2}-\delta\right) \sigma\left(t-c+3 v_{1}+2 v_{2}\right)}{\sigma\left(t+v_{1}\right) \sigma\left(t+v_{1}+v_{2}\right) \sigma\left(t+2 v_{1}+v_{2}\right) \sigma\left(t+2 v_{1}+2 v_{2}\right)},
\end{aligned}
$$

where $2\left(v_{1}+v_{2}\right) \equiv 0$ and

$$
\begin{aligned}
\rho_{1} & =\frac{\sigma(c) \sigma\left(c-v_{1}\right) \sigma\left(-c+v_{1}+v_{2}\right) \sigma\left(-c+2 v_{1}+v_{2}\right)}{\varepsilon \sigma\left(-2 c+2 v_{1}+v_{2}\right) \sigma\left(v_{1}+v_{2}\right) \sigma\left(v_{1}+v_{2}-\delta\right) \sigma\left(2 a+v_{2}-\delta\right)}, \\
\rho_{2} & =\frac{\sigma(a) \sigma\left(a+v_{2}\right) \sigma\left(-a+v_{1}\right) \sigma\left(-a+v_{1}+v_{2}\right)}{\varepsilon \sigma\left(-2 a+v_{1}\right) \sigma\left(v_{1}+v_{2}\right) \sigma\left(v_{1}+v_{2}-\delta\right) \sigma\left(2 c-v_{1}-\delta\right)} .
\end{aligned}
$$

Thus, for any initial point $x$, its iterations $f^{n}(x)$ are given by the above formulas with $t=n \delta+t_{0}$, with a suitable lattice of periods and suitable parameters $v_{1}, v_{2}, \delta, t_{0} \in \mathbb{C}$ (where, recall , $2\left(v_{1}+\right.$ $\left.\left.v_{2}\right) \equiv 0\right)$. The constants $a$ and $c$ are defined by (65)-(66).

Proof. We show how to verify (54). The remaining three equations may be dealt with in the same way. Under conditions (65)-(66) the function $1+\varepsilon\left(x_{3}-x_{1}\right)$ has the zeros $-c,-d$, $-c-v_{1}-v_{2}+\delta,-d-v_{1}-v_{2}+\delta$, while the function $1-\varepsilon\left(x_{3}-x_{1}\right)$ has the zeros $-c+\delta$, $-d+\delta,-c-v_{1}-v_{2},-d-v_{1}-v_{2}$. Hence, with the help of the periodicity condition $2\left(v_{1}+v_{2}\right) \equiv 0$, it is easy to see that there holds

$$
\begin{aligned}
1 & +\varepsilon\left(x_{3}-x_{1}\right)= \\
& =C_{1} \frac{\sigma(t+c) \sigma\left(t-c+\delta+2 v_{1}+v_{2}\right) \sigma\left(t+c-\delta+v_{1}+v_{2}\right) \sigma\left(t-c+3 v_{1}+2 v_{2}\right)}{\sigma\left(t+v_{1}\right) \sigma\left(t+v_{1}+v_{2}\right) \sigma\left(t+2 v_{1}+v_{2}\right) \sigma\left(t+2 v_{1}+2 v_{2}\right)}
\end{aligned}
$$

as well as

$$
\begin{aligned}
1 & -\varepsilon\left(x_{3}-x_{1}\right)= \\
& =C_{2} \frac{\sigma\left(t+c+v_{1}+v_{2}\right) \sigma\left(t-c+\delta+3 v_{1}+2 v_{2}\right) \sigma(t+c-\delta) \sigma\left(t-c+2 v_{1}+v_{2}\right)}{\sigma\left(t+v_{1}\right) \sigma\left(t+v_{1}+v_{2}\right) \sigma\left(t+2 v_{1}+v_{2}\right) \sigma\left(t+2 v_{1}+2 v_{2}\right)}
\end{aligned}
$$

with some constants $C_{1}, C_{2}$ depending on $v_{1}, v_{2}, c, \varepsilon$. With the help of the three-term identity for the $\sigma$-function (38) we see that the difference $x_{3}-x_{1}$ has a zero at $t=-v_{1}-v_{2} / 2$. We therefore determine $C_{1}$ and $C_{2}$ by setting $t=-v_{1}-v_{2} / 2$ in (67) and (68), giving

$$
\begin{aligned}
& C_{1}=\frac{\sigma^{2}\left(v_{2} / 2\right) \sigma\left(v_{1}+v_{2} / 2\right) \sigma\left(v_{1}+3 v_{2} / 2\right)}{\sigma\left(v_{1}+v_{2} / 2-c\right) \sigma\left(v_{1}+v_{2} / 2-c+\delta\right) \sigma\left(v_{2} / 2+c-\delta\right) \sigma\left(2 v_{1}+3 v_{2} / 2-c\right)}, \\
& C_{2}=\frac{\sigma^{2}\left(v_{2} / 2\right) \sigma\left(v_{1}+v_{2} / 2\right) \sigma\left(v_{1}+3 v_{2} / 2\right)}{\sigma\left(v_{2} / 2+c\right) \sigma\left(2 v_{1}+3 v_{2} / 2+\delta-c\right) \sigma\left(v_{1}+v_{2} / 2-c+\delta\right) \sigma\left(v_{1}+v_{2} / 2-c\right)} .
\end{aligned}
$$

Hence,

$$
\frac{C_{1}}{C_{2}}=\frac{\sigma\left(v_{2} / 2+c\right) \sigma\left(2 v_{1}+3 v_{2} / 2+\delta-c\right)}{\sigma\left(v_{2} / 2+c-\delta\right) \sigma\left(2 v_{1}+3 v_{2} / 2-c\right)}
$$


Now, by virtue of $(67)-(68)$, equation of motion (54) takes the form

$$
\begin{aligned}
& \frac{\sigma\left(t+c+2 v_{1}+2 v_{2}\right) \sigma\left(t-c+\delta+2 v_{1}+v_{2}\right)}{\sigma\left(t+c+v_{1}+v_{2}\right) \sigma\left(t+\delta-c+3 v_{1}+2 v_{2}\right)} \\
& \quad=\frac{C_{1}}{C_{2}} \frac{\sigma(t+c) \sigma\left(t-c+\delta+2 v_{1}+v_{2}\right) \sigma\left(t+c+2 v_{1}+2 v_{2}-\delta\right)}{\sigma\left(t+c+v_{1}+v_{2}\right) \sigma\left(t+\delta-c+3 v_{1}+2 v_{2}\right) \sigma(t+c-\delta)}
\end{aligned}
$$

which reduces to

$$
\frac{C_{1}}{C_{2}}=\frac{\sigma\left(t+c+2 v_{1}+2 v_{2}\right) \sigma(t+c-\delta)}{\sigma(t+c) \sigma\left(t+c+2 v_{1}+2 v_{2}-\delta\right)}
$$

but this identity is easily verified using (69) and the quasi-periodicity of the $\sigma$-function.

\section{CONCLusions}

We have shown how to use Hirota-Kimura bases to construct explicit solutions of birational maps which are solvable in terms of elliptic functions. The appealing features of this approach are the following:

- It is systematic: Ansätze for explicit solutions are derived via searching for algebraic curves on which different two-dimensional projections of the orbits lie.

- We do not look for or try to construct additional integrable structures (for instance Lax pairs), a process which would usually require large amounts of guesswork and/or research experience.

It would be interesting to apply this approach to further birational maps which can be solved in terms of elliptic functions. These are, basically, almost all Hirota-Kimura discretizations presented in [13], with the exception of the discrete Clebsch system (which is likely to admit solutions in terms of theta functions of genus 2 Riemann surfaces), and include, among others, the discrete Lagrange top and the discrete Kirchhoff system.

\section{ACKNOWLEDGMENTS}

This research was supported by the DFG Collaborative Research Center TRR 109 "Discretization in Geometry and Dynamics". We would like to thank the referee for valuable remarks.

\section{APPENDiX A. SOLUTION TO THE INITIAL VALUE PROBLEM FOR THE DISCRETE VOLTERRA CHAIN WITH $N=3$ \\ Yuri Fedorov 11}

Here we provide an alternative construction of explicit sigma-function solutions for the map $\mathrm{dVC}_{3}$ given by eq. (30), namely,

$$
\begin{aligned}
& \widetilde{x}_{1}-x_{1}=\varepsilon x_{1}\left(\widetilde{x}_{2}-\widetilde{x}_{3}\right)+\varepsilon \widetilde{x}_{1}\left(x_{2}-x_{3}\right), \\
& \widetilde{x}_{2}-x_{2}=\varepsilon x_{2}\left(\widetilde{x}_{3}-\widetilde{x}_{1}\right)+\varepsilon \widetilde{x}_{2}\left(x_{3}-x_{1}\right), \\
& \widetilde{x}_{3}-x_{3}=\varepsilon x_{3}\left(\widetilde{x}_{1}-\widetilde{x}_{2}\right)+\varepsilon \widetilde{x}_{3}\left(x_{1}-x_{2}\right) .
\end{aligned}
$$

\footnotetext{
${ }^{1}$ Department of Mathematics, Politechnic University of Catalonia, Barcelona, Spain. E-mail: Yuri.Fedorov@upc.edu
} 
Our method, like the method of the main paper, is based on an extensive use of the symbolic manipulation software Maple. Recall that the map $\mathrm{dVC}_{3}$ possesses the conserved quantities given by eq. (31),

$$
H_{1}(x)=x_{1}+x_{2}+x_{3}, \quad H_{2}(x, \varepsilon)=\frac{x_{1} x_{2} x_{3}}{1-\varepsilon^{2}\left(x_{1}^{2}+x_{2}^{2}+x_{3}^{2}-2 x_{1} x_{2}-2 x_{2} x_{3}-2 x_{3} x_{1}\right)},
$$

which implies that the complex invariant manifold $\left\{x \in \mathbb{C}^{3}: H_{1}(x)=H_{1}, H_{2}(x)=H_{2}\right\}$ of the map is the intersection of a cubic surface and a plane, and, therefore, in general, is an open subset of an elliptic curve. For fixed constants of motion $H_{1}, H_{2}$, we eliminate $x_{3}$ from the integrals (71) and get the equation of the planar elliptic curve $E$ :

$$
f\left(x_{1}, x_{2}\right)=1-\varepsilon^{2}\left(H_{1}^{2}-4 x_{1} x_{2}-4\left(H_{1}-x_{1}-x_{2}\right)\left(x_{1}+x_{2}\right)\right)-\frac{x_{1} x_{2}\left(H_{1}-x_{1}-x_{2}\right)}{H_{2}}=0 .
$$

Then we apply the classical algorithm of parameterization of elliptic curves embedded in $\mathbb{C}^{2}$, implemented in the Maple command Weierstrassform $\left(f, x_{1}, x_{2}, X, Y\right)$. It immediately returns a canonical form of this curve:

$$
Y^{2}=P_{3}(X)=\frac{1}{4}\left(4 X^{3}-g_{2} X-g_{3}\right)
$$

where

$$
\begin{aligned}
g_{2}= & \frac{1}{12}\left(H_{1}-12 \varepsilon^{2} H_{2}\right)\left(H_{1}^{3}-24 H_{2}-12 \varepsilon^{2} H_{1}^{2} H_{2}+48 \varepsilon^{4} H_{1} H_{2}^{2}-192 \varepsilon^{6} H_{2}^{3}\right), \\
g_{3}= & -H_{2}^{2}+\frac{1}{6} H_{1}^{3} H_{2}-\frac{1}{216} H_{1}^{6}-4 \varepsilon^{2} H_{1}^{2} H_{2}^{2}+\frac{1}{6} \varepsilon^{2} H_{1}^{5} H_{2}-\frac{7}{3} \varepsilon^{4} H_{1}^{4} H_{2}^{2}+40 \varepsilon^{4} H_{1} H_{2}^{3} \\
& -160 \varepsilon^{6} H_{2}^{4}+\frac{40}{3} \varepsilon^{6} H_{1}^{3} H_{2}^{3}-256 \varepsilon^{10} H_{1} H_{2}^{5}+512 \varepsilon^{12} H_{2}^{6} .
\end{aligned}
$$

The same Maple command also gives the original variables $x_{1}, x_{2}$ in terms of $X, Y$ :

$$
\begin{aligned}
& x_{1}=\frac{H_{1}^{3}-12 H_{2}-16 \varepsilon^{2} H_{1}^{2} H_{2}+48 \varepsilon^{4} H_{1} H_{2}^{2}+192 \varepsilon^{6} H_{2}^{3}-24 Y-12 B X}{2\left(b^{2}-12 X\right)}, \\
& x_{2}=\frac{4 H_{2}\left(3-2 \varepsilon^{2} H_{1}^{2}+24 \varepsilon^{4} H_{1} H_{2}-48 \varepsilon^{6} H_{2}^{2}\right)-48 \varepsilon^{2} H_{2} X}{b^{2}-12 X},
\end{aligned}
$$

where the following abbreviations are used:

$$
b=H_{1}-12 \varepsilon^{2} H_{2}, \quad B=H_{1}-4 \varepsilon^{2} H_{2} .
$$

Then the relation $x_{1}+x_{2}+x_{3}=H_{1}$ yields

$$
x_{3}=\frac{H_{1}^{3}-12 H_{2}-16 \varepsilon^{2} H_{1}^{2} H_{2}+48 \varepsilon^{4} H_{1} H_{2}^{2}+192 \varepsilon^{6} H_{2}^{3}+24 Y-12 B X}{2\left(b^{2}-12 X\right)} .
$$

The standard parametrization of the Weierstrass canonical form of $E$ is:

$$
X=\wp\left(u ; g_{2}, g_{3}\right), \quad Y=\frac{1}{2} \wp^{\prime}\left(u ; g_{2}, g_{3}\right), \quad u \in \mathbb{C} .
$$

To obtain the parameterization of $E$ in terms of sigma-functions of $u$, we note that near the only infinite point $\infty$ of $E$ we have the expansions

$$
X=1 / u^{2}+O(u), \quad Y=-1 / u^{3}+O(1) .
$$


Then the above expressions $x_{i}(X, Y)$ imply that $x_{2}(u)$ has a pair of simple poles at $u= \pm v$, with

$$
\wp(v)=\frac{b^{2}}{12}, \quad \wp^{\prime}(v)=-H_{2}\left(1+\varepsilon H_{1}-8 \varepsilon^{3} H_{2}\right)\left(1-\varepsilon H_{1}+8 \varepsilon^{3} H_{2}\right),
$$

and a pair of simple zeros at $u= \pm \delta$ with

$$
\begin{aligned}
& \wp(\delta)=\frac{1}{4 \varepsilon^{2}}-\frac{1}{6}\left(H_{1}^{2}-12 \varepsilon^{2} H_{1} H_{2}+24 \varepsilon^{2} H_{2}^{2}\right), \\
& \wp^{\prime}(\delta)=-\frac{1}{4 \varepsilon^{3}}\left(1+\varepsilon H_{1}-8 \varepsilon^{3} H_{2}\right)\left(1-\varepsilon H_{1}+8 \varepsilon^{3} H_{2}\right) .
\end{aligned}
$$

Hence, we have a parametrization in terms of the sigma function $\sigma\left(u ; g_{2}, g_{3}\right)$ :

$$
x_{2}(u)=\rho_{2} \frac{\sigma(u-\delta) \sigma(u+\delta)}{\sigma(u-v) \sigma(u+v)}
$$

with a constant factor $\rho_{2}$, which is calculated from the condition

$$
x_{2}(u=0)=\rho_{2} \frac{\sigma^{2}(\delta)}{\sigma^{2}(v)}=\lim _{X \rightarrow \infty} x_{2}(X)=4 \varepsilon^{2} H_{2} .
$$

Next, in view of (79) the denominator of $x_{1}(X, Y)$ in (76) has simple zeros at $u= \pm v$ and a double pole at $u=0$. The numerator has a triple pole at $u=0$. The zeros of the numerator are defined by the relation

$$
Y=-\frac{B}{2} X+\frac{1}{24} H_{1}^{3}-\frac{1}{2} H_{2}-\frac{2}{3} \varepsilon^{2} H_{1}^{2} H_{2}+2 \varepsilon^{4} H_{1} H_{2}^{2}+8 \varepsilon^{6} H_{2}^{3} .
$$

Substituting this into the canonical equation (72), we get the following condition for $X$ :

$$
\left(12 X-b^{2}\right)\left(12 X-H_{1}^{2}-24 \varepsilon H_{2}+48 \varepsilon^{4} H_{2}^{2}\right)\left(12 X-H_{1}^{2}+24 \varepsilon H_{2}+48 \varepsilon^{4} H_{2}^{2}\right)=0 .
$$

The latter condition in combination with (83) defines 3 points on the curve $E$. One concludes that the numerator vanishes at $u=v, \mu_{1}, \mu_{2}$ with

$$
\begin{aligned}
& \wp\left(\mu_{1}\right)=\frac{1}{12} H_{1}^{2}+2 \varepsilon H_{2}-4 \varepsilon^{4} H_{2}^{2}, \quad \wp^{\prime}\left(\mu_{1}\right)=-H_{2}\left(1+\varepsilon H_{1}\right)\left(1+\varepsilon H_{1}-8 \varepsilon^{3} H_{2}\right), \\
& \wp\left(\mu_{2}\right)=\frac{1}{12} H_{1}^{2}-2 \varepsilon H_{2}-4 \varepsilon^{4} H_{2}^{2}, \quad \wp^{\prime}\left(\mu_{2}\right)=-H_{2}\left(1-\varepsilon H_{1}\right)\left(1-\varepsilon H_{1}+8 \varepsilon^{3} H_{2}\right) .
\end{aligned}
$$

Combining these observation, we conclude that $x_{1}$ has simple poles at $u=0,-v$ and simple zeros at $u=\mu_{1}, \mu_{2}$ and no zeros or poles elsewhere. As a result, one can write

$$
x_{1}(u)=\rho_{1} \frac{\sigma\left(u-\mu_{1}\right) \sigma\left(u-\mu_{2}\right)}{\sigma(u) \sigma(u+v)},
$$

with a suitable constant factor $\rho_{1}$. The expressions $x_{1}(X, Y), x_{3}(X, Y)$ differ only by sign of $Y$, hence $x_{3}(u)=x_{1}(-u)$, that is,

$$
x_{3}(u)=\rho_{1} \frac{\sigma\left(u+\mu_{1}\right) \sigma\left(u+\mu_{2}\right)}{\sigma(u) \sigma(u-v)} .
$$

It follows that $\mu_{1}+\mu_{2} \equiv-v$, where $\equiv$ denotes equality modulo the period lattice of $E$. Thus, we arrive at

$$
x_{1}(u)=\rho_{1} \frac{\sigma\left(u-\mu_{1}\right) \sigma\left(u+\mu_{1}+v\right)}{\sigma(u) \sigma(u+v)}, \quad x_{3}(u)=\rho_{1} \frac{\sigma\left(u-\mu_{1}-v\right) \sigma\left(u+\mu_{1}\right)}{\sigma(u) \sigma(u-v)} .
$$


Since $x_{1}, x_{2}, x_{3}$ enter into the integrals $H_{1}(x), H_{2}(x)$ in a symmetric way, there is a cyclic group acting on $E$ by translations:

$$
x_{1}=f(u), \quad x_{2}=f(u+h), \quad x_{3}=f(u+2 h), \quad x_{1}=f(u+3 h), \quad 3 h \equiv 0 .
$$

The above formulas for $x_{i}(u)$ are compatible with equation $(86)$ if and only if $h=v$ and $\delta= \pm\left(v-\mu_{1}\right)$. By a straightforward computation, one shows that

$$
\left|\begin{array}{ccc}
\wp(v) & \wp^{\prime}(v) & 1 \\
\wp(\delta) & -\wp^{\prime}(\delta) & 1 \\
\wp\left(\mu_{1}\right) & -\wp^{\prime}\left(\mu_{1}\right) & 1
\end{array}\right|=0
$$

which, according to the classical addition theorem for the $\wp$-function, yields $\delta=v-\mu_{1}$. As a consequence, we can write

$$
\begin{aligned}
& x_{1}(u)=\rho_{1} \frac{\sigma(u+\delta-v) \sigma(u-\delta+2 v)}{\sigma(u) \sigma(u+v)}, \\
& x_{2}(u)=\rho_{2} \frac{\sigma(u+\delta) \sigma(u-\delta)}{\sigma(u+v) \sigma(u-v)}, \\
& x_{3}(u)=\rho_{1} \frac{\sigma(u+\delta-2 v) \sigma(u-\delta+v)}{\sigma(u) \sigma(u-v)} .
\end{aligned}
$$

By shifting the argument $u$ by the period $3 v$ in some terms above, these relations take the form (86):

$$
\begin{aligned}
& x_{1}(u)=\bar{\rho} \frac{\sigma(u-\delta-v) \sigma(u+\delta-v)}{\sigma(u) \sigma(u-2 v)}, \\
& x_{2}(u)=\bar{\rho} \frac{\sigma(u-\delta) \sigma(u+\delta)}{\sigma(u+v) \sigma(u-v)}, \\
& x_{3}(u)=\bar{\rho} \frac{\sigma(u-\delta+v) \sigma(u+\delta+v)}{\sigma(u+2 v) \sigma(u)},
\end{aligned}
$$

where $\bar{\rho}=\rho_{2}=4 \varepsilon^{2} H_{2} \frac{\sigma^{2}(v)}{\sigma^{2}(\delta)}$ and, as follows from the above, $v=\int_{\infty}^{b^{2} / 12} \frac{d X}{2 \sqrt{P_{3}(X)}}$.

Observe that, like in the continuous case, the condition $3 v \equiv 0$ can be verified analytically. Namely, using (80), (73), we compute: $\wp^{\prime \prime}(v)=6 \wp^{2}(v)-g_{2} / 2=-b \wp^{\prime}(v)$. Then the classical duplication formula for $\wp(v)$ yields

$$
\wp(2 v)=\frac{1}{4}\left(\frac{\wp^{\prime \prime}(v)}{\wp^{\prime}(v)}\right)^{2}-2 \wp(v)=\frac{b^{2}}{4}-2 \wp(v)=3 \wp(v)-2 \wp(v)=\wp(v) .
$$

Since $v \neq 0$, we find: $2 v \equiv-v$, so that $3 v \equiv 0$.

Given the values of the coordinates $x_{1}, x_{2}, x_{3}$, and therefore the corresponding constants $H_{1}, H_{2}, \delta, v$, the value of the parameter $u$ can be recovered from equation (76), which yields

$$
X=\frac{x_{2} \wp(v)-4 \varepsilon^{2} H_{2} \wp(\delta)}{x_{2}-4 \varepsilon^{2} H_{2}},
$$

so that, up to sign, $u=\int_{\infty}^{X} \frac{d X^{\prime}}{2 \sqrt{P_{3}\left(X^{\prime}\right)}}$. The sign of $u$ can be determined by verifying, say, the first expression in (88) with the given $x_{1}$. 
$\mathrm{dVC}_{3}$ as a translation on $E$. Since the map $(70)$ is algebraic, its action on $E$ must be a translation by a constant vector. We show that the following result holds true.

Theorem 10. The shift on E generated by the map (70) is equal to $\delta$, so that the orbits of $d V C_{3}$ $x(n)=f^{n}(x(0))$ are given by the expressions

$$
\begin{aligned}
& x_{1}(n)=\bar{\rho} \frac{\sigma\left(u_{n}-\delta-v\right) \sigma\left(u_{n}+\delta-v\right)}{\sigma\left(u_{n}\right) \sigma\left(u_{n}-2 v\right)}, \\
& x_{2}(n)=\bar{\rho} \frac{\sigma\left(u_{n}-\delta\right) \sigma\left(u_{n}+\delta\right)}{\sigma\left(u_{n}+v\right) \sigma\left(u_{n}-v\right)}, \\
& x_{3}(n)=\bar{\rho} \frac{\sigma\left(u_{n}-\delta+v\right) \sigma\left(u_{n}+\delta+v\right)}{\sigma\left(u_{n}+2 v\right) \sigma\left(u_{n}\right)},
\end{aligned}
$$

where $u_{n}=n \delta+u_{0}$, and $v, \delta$ are specified in (80), (81). The phase $u_{0}$ is determined from the initial values of $x_{1}, x_{2}, x_{3}$ as described above.

Proof. Substitute the expressions $x_{i}(X, Y), i=1,2,3$ into (70) and obtain, up to a constant factor,

where

$$
\widetilde{x}_{1}=x_{1} \frac{(X-\wp(v)) U(X, Y)}{\left(X-\wp\left(\mu_{1}\right)\right)\left(X-\wp\left(\mu_{2}\right)\right)(X-\wp(\delta))}
$$

$$
\begin{aligned}
U(X, Y)= & 12\left(1-\varepsilon H_{1}+12 \varepsilon^{3} H_{2}+4 \varepsilon^{4} H_{1} H_{2}\right) X+24 \varepsilon\left(1-\varepsilon H_{1}\right) Y \\
& -H_{1}^{2}+\varepsilon H_{1}^{3}-36 \varepsilon H_{2}+12 \varepsilon^{2} H_{1} H_{2}-144 \varepsilon^{4} H_{2}^{2}+8 \varepsilon^{4} H_{1}^{3} H_{2} \\
& -144 \varepsilon^{5} H_{1} H_{2}^{2}-96 \varepsilon^{6} H_{1}^{2} H_{2}^{2}+576 \varepsilon^{7} H_{2}^{3}+192 \varepsilon^{8} H_{1} H_{2}^{3} .
\end{aligned}
$$

In view of equation (72), on $E$ the function $U(X, Y)$ has simple zeros at $u=-\mu_{1}, u=\delta$, and at a point $u=q$ with

$$
\begin{gathered}
\wp(q)=\frac{1}{12}\left(H_{1}^{2}-2 \varepsilon H_{1}^{3}+48 \varepsilon H_{2}+\varepsilon^{2} H_{1}^{4}-24 \varepsilon^{2} H_{1} H_{2}-24 \varepsilon^{4} H_{1}^{3} H_{2}+528 \varepsilon^{4} H_{2}^{2}\right. \\
\left.+96 \varepsilon^{5} H_{1} H_{2}^{2}+144 \varepsilon^{6} H_{1}^{2} H_{2}^{2}\right) /\left(1-\varepsilon H_{1}\right)^{2}, \\
\wp^{\prime}(q)=-H_{2}\left(1-\varepsilon H_{1}+8 \varepsilon^{3} H_{2}\right)\left(1+2 \varepsilon H_{1}-2 \varepsilon^{3} H_{1}^{3}+72 \varepsilon^{3} H_{2}-\varepsilon^{4} H_{1}^{4}+24 \varepsilon^{4} H_{1} H_{2}\right. \\
\left.+24 \varepsilon^{5} H_{1}^{2} H_{2}+8 \varepsilon^{6} H_{1}^{3} H_{2}\right) /\left(1-\varepsilon H_{1}\right)^{3} .
\end{gathered}
$$

On the other hand, $U(X, Y)$ has a triple pole at $u=0$. Then (91) implies that the function $\widetilde{x}_{1}(u) / x_{1}(u)$ has a simple zero at $X=\infty$, that is, at $u=0$. Summarizing, we have:

$$
\widetilde{x}_{1}(u) \sim x_{1}(u) \frac{\sigma(u) \sigma(u-v) \sigma(u+v) \sigma(u-q)}{\sigma(u+\delta) \sigma\left(u-\mu_{1}\right) \sigma\left(u-\mu_{2}\right) \sigma\left(u+\mu_{2}\right)},
$$

where " $\sim$ " means equality up to a constant factor. Recall that $\mu_{1}+\mu_{2}=-v$ and $\mu_{1}=$ $v-\delta$, so that $\mu_{2}=\delta-2 v$. In view of (85),

we find:

$$
x_{1}(u) \sim \frac{\sigma\left(u-\mu_{1}\right) \sigma\left(u-\mu_{2}\right)}{\sigma(u) \sigma(u+v)}=\frac{\sigma(u-v+\delta) \sigma(u+2 v-\delta)}{\sigma(u) \sigma(u+v)},
$$

$$
\widetilde{x}_{1}(u) \sim \frac{\sigma(u-v) \sigma(u-q)}{\sigma(u+\delta) \sigma\left(u+\mu_{2}\right)}=\frac{\sigma(u-v) \sigma(u-q)}{\sigma(u+\delta) \sigma(u+\delta-2 v)} \sim \frac{\sigma(u+2 v) \sigma(u-q)}{\sigma(u+\delta) \sigma(u+v+\delta)} .
$$


By comparing the poles of $x_{1}(u)$ and $\widetilde{x}_{1}(u)$, we conclude that the translation on $E$ is given by $u \mapsto u+\delta$.

Expressions (88) and the result of Theorem 10 confirm formulas (32)-(34) of the main body of the paper, and provide us with the further information, like the expressions of the Weierstrass invariants $g_{2}, g_{3}$ and of the shift $\delta$ through the integrals of motion $H_{1}$, $\mathrm{H}_{2}$. Observe that in the continuous limit $\varepsilon \rightarrow 0$ the invariants (73), (74) tend to (28), and similarly expressions (80) tend to $\wp(v)=H_{1}^{2} / 12, \wp^{\prime}(v)=-H_{2}$, which coincide with (29). Moreover, according to (81) and the asymptotical behavior of $\wp(u)$ near zero, for small $\varepsilon$ one has the expansion

$$
\delta=2 \varepsilon+O\left(\varepsilon^{2}\right) .
$$

Real orbits of $\mathbf{d} \mathbf{V C}_{3}$. Let $\omega_{1}, \omega_{3}$ be half-periods of the elliptic curve $E \in \mathbb{C}^{3}$, and $\omega_{1} \in \mathbb{R}$. Depending on the values $H_{1}, H_{2}$, the polynomial $P_{3}(X)$ in $(72)$ can have one or 3 real roots. Then the real part $E_{R}$ of $E$ has one, respectively two connected components. In the parallelogram of periods they are given by segments parallel to the real axis in the complex plane $u$. In the second case one can choose the second half-period $\omega_{3}$ to be imaginary, and the two segments are $\left\{2 \omega_{1} t: t \in[0,1]\right\}$ and $\left\{2 \omega_{1} t+\omega_{3}: t \in[0,1]\right\}$. Observe that, according to (80), the 1/3-period $v$ is always real.

Next, as follows from (93), for sufficiently small $\varepsilon$, the shift $\delta$ is also small and real, hence the $\mathrm{dVC}_{3}$-orbit of an initial point on $E_{R}$ belongs to the same connected component of $E_{R}$. The situation is different for relatively big $\varepsilon$, when $\delta$ becomes a sum of a real number and the imaginary half-period $\omega_{3}$. Then the point $\left(x_{1}, x_{2}, x_{3}\right)$ changes the component of $E_{R}$ under each iteration of the map.

For some special values of $H_{1}, H_{2}, \varepsilon$ satisfying $\varepsilon\left(H_{1}-8 \varepsilon^{2} H_{2}\right)= \pm 1$ the polynomial $P_{3}(X)$ has a double root which coincides with the expression for $\wp(\delta)$ in (81). In this case the invariant curve $\left\{x \in \mathbb{C}^{3}: H_{1}(x)=H_{1}, H_{2}(x)=H_{2}\right\}$ becomes rational and, as one can show, the orbit $\mathcal{O}(x)$ consists of just two points on it.

Numerical examples. Choosing the initial point $\left(x_{1}, x_{2}, x_{3}\right)=(3,4,5)$ and $\varepsilon=1$, we obtain $H_{1}=12, H_{2}=4 / 3$, and the curve $E$ in the Weierstrass form is

$$
Y^{2}=X^{3}-\frac{88}{27} X+\frac{1636}{729} \text { with } g_{2}=\frac{352}{27}, g_{3}=-\frac{6544}{729} .
$$

Up to $10^{-8}$, the $X$-coordinates of the branch points are

$$
e_{1}=-2.0825183, \quad e_{2}=0.9600348, \quad e_{3}=1.12248349,
$$

and the real and imaginary half-periods of $E$ are, respectively, $\omega_{1}=1.62107698$ and $\omega_{3}=0.88886315 i$. The real part $E_{R} \subset \mathbb{R}^{3}$ consists of two connected components, one is compact, whereas the other one is not.

Next, following (80), (81), one has $\wp(v)=4 / 3, \wp(\delta)=41 / 36$, and the Abel map gives

$$
v=\int_{\infty}^{\wp(v)} \frac{d X}{Y}=1.08084313, \quad \delta=\int_{\infty}^{\wp(\delta)} \frac{d X}{Y}=1.44663208
$$

Observe that $v=2 \omega_{1} / 3$ (up to $10^{-7}$ ). Further, the constant $\bar{\rho}$ in $(88)$ equals $\bar{\rho}=3.71953594$ and, according to $(89)$, the initial phase equals $u_{0}=1.265142+\omega_{3}$. Then the evaluation of expressions (88) with $u=u_{0}$ with Maple function WeierstrassSigma $\left(u, g_{2}, g_{3}\right)$ recovers the initial values $\left(x_{1}, x_{2}, x_{3}\right)=(3,4,5)$ up to $10^{-6}$. 
Finally, the first iteration of the map (70) yields

$$
x_{1}(1)=71 / 15, \quad x_{2}(1)=212 / 45, \quad x_{3}(1)=23 / 9,
$$

and the formulas $(90)$ for $n=1$ give the same values up to $10^{-4}$.

In the second example we take the initial point $\left(x_{1}, x_{2}, x_{3}\right)=(3,-4,5)$, and again $\varepsilon=1$, which give $H_{1}=4, H_{2}=60 / 83$, and the curve $E$ in the canonical form reads

$$
Y^{2}=X^{3}-\frac{892144696}{142374963} X+\frac{52898505488012}{8827390080963} .
$$

The branch points ordered according to $e_{1}<e_{2}<e_{3}$ are

$$
e_{1}=-2.8880922, \quad e_{2}=1.342284391, \quad e_{3}=1.54580078 .
$$

As in the previous example, $E_{R}$ consists of two connected components. Following (80), (81), one finds $\wp(v)=1.882107, \wp(\delta)=1.27617$. Without further calculations, we observe that, whereas $\wp(v)>e_{3}$ and therefore $v \in \mathbb{R}$, one has $\wp(\delta) \in\left(e_{1}, e_{2}\right)$, hence the shift $\delta$ is the sum of a real number and the imaginary half-period $\omega_{3}$. As was mentioned above, in this case an initial point and its image under the map (70) belong to different components of $E_{R}$.

Acknowledgments. The author acknowledges support of the Spanish MINECO-FEDER Grant MTM2012-37070. He is also grateful to Yu. Suris for useful discussions.

\section{REFERENCES}

[1] R.J. Baxter, Exactly solved models in statistical mechanics, Academic Press, London, 1989.

[2] E. Celledoni, R.I. McLachlan, B. Owren, G.R.W.Quispel, Geometric properties of Kahan's method, J. Phys. A: Math. Theor., 46 (2013) 025201 (12 pp).

[3] E. Celledoni, R.I. McLachlan, D.I. McLaren, B. Owren, G.R.W.Quispel, Integrability properties of Kahan's method, J. Phys. A: Math. Theor., 47 (2014) 365202 (20 pp).

[4] G.H. Halphen, Traité des fonctions elliptiques et de leurs applications, Gauthier-Villars, Paris, 1888.

[5] R. Hirota, K. Kimura, Discretization of the Euler top, J. Phys. Soc. Japan, 69, Nr. 3 (2000), 627-630.

[6] A.N.W. Hone, M. Petrera, Three-dimensional discrete systems of Hirota-Kimura type and deformed Lie-Poisson algebras, J. Geom. Mech., 1, Nr. 1 (2009), 55-85.

[7] M. Kac, P. van Moerbecke, On an explicitly soluble system of non-linear differential equations related to certain Toda lattices, Adv. Math., 16 (1975), 160-169.

[8] W. Kahan, Unconventional numerical methods for trajectory calculations, Unpublished lecture notes, 1993.

[9] K. Kimura, R. Hirota, Discretization of the Lagrange top, J. Phys. Soc. Japan, 69, Nr. 10 (2000), 3193-3199.

[10] A.V. Kitaev, A note on the averaging for single-phase elliptic solutions of the Toda and Volterra lattices, Physica D, 74 (1994), 45-58.

[11] S.V. Manakov, On the complete integrability and stochastization in discrete dynamical systems, Zh. Exp. Theor. Phys. 67 (1974), 543-555.

[12] M. Petrera, A. Pfadler, Yu.B. Suris, On integrability of Hirota-Kimura-type discretizations: experimental study of the discrete Clebsch system, Exp. Math., 18, Nr. 2 (2009), 223-247.

[13] M. Petrera, A. Pfadler, Yu.B. Suris, On integrability of Hirota-Kimura-type discretizations, Reg. Chaotic Dyn. 16, Nr. 3/4 (2011), 245-289.

[14] M. Petrera, Yu.B. Suris, On the Hamiltonian structure of Hirota-Kimura discretization of the Euler top, Math. Nachr. 283, Nr. 11 (2010), 1654-1663.

[15] V.L. Vereshchagin, Hamiltonian structure of averaged difference systems, Math. Zametki, 44, Nr. 5 (1988), 584-595.

[16] A.P. Veselov, Integration of the stationary problem for a classical spin chain, Theor. Math. Phys., 71, Nr. 1 (1987), 154-159.

[17] M. Van Hoeij, An algorithm for computing the Weierstrass normal form - In: ISSAC' 95 Proceedings, 1995. 
[18] E.T. Whittaker, G.N. Watson, A course of modern analysis, Cambridge University Press, Cambridge, 1996. 